\title{
Reproducing the pressure-time signature of membrane filtration: The interplay between fouling, caking, and elasticity
}

\author{
J. G. Herterich ${ }^{\mathrm{a}, \mathrm{b}}$, I. M. Griffiths ${ }^{\mathrm{b}}$, D. Vella ${ }^{\mathrm{b}}$ \\ ${ }^{a}$ School of Mathematics and Statistics, University College Dublin, Belfield, Dublin 4, Ireland \\ ${ }^{b}$ Mathematical Institute, University of Oxford, Radcliffe Observatory Quarter, Oxford OX2 6GG, United Kingdom
}

\begin{abstract}
We develop a mathematical model of direct-flow filtration operating at constant flux to understand the pressure-time signature. We combine fluid flow with membrane fouling and caking to explain the gradual increase in driving pressure that is often reported. We model the periodic backflushes used to clean such membranes and show that the elasticity of the membrane may explain the limited effectiveness of these backflushes. We also consider strategies for the operation of direct-flow filtration and show that tuning the flux and rate of backflushing, as well as the membrane material structure, may allow for improvements in membrane performance.
\end{abstract}

Keywords: Filtration, Fouling, Caking, Backflushing, Elasticity, Mathematical modelling

\section{Introduction}

In the constant-flux membrane filtration of liquid, the required driving pressure is known to increase over time $[1,2,3,4]$. This increase is attributed to the particles that are removed by the filter, which cause fouling and caking, that is, the trapping of particles within and on the membrane, respectively. One way of mitigating this increase in driving pressure, and hence to extend the lifetime and efficiency of a membrane, is to perform a 'backflush' at regular intervals: the direction of the fluid flux is briefly reversed, removing the cake layer and decreasing the driving pressure required for the next period of (forwards) filtration. However, experiments show that backflushing at regular intervals does not completely reduce the driving pressure back to its value at the start of filtration [2,4]. A schematic illustration of the pressure versus time trace, Figure 2, shows that the driving pressure immediately following each successive backflush gradually increases and, further, that the increase in driving pressure during each subsequent filtration cycle grows. As a result, the filtration process becomes less efficient with time. The analogue of this pressure-time signature when operating at constant pressure has also been reported. In this case, the flux achieved at given pressure gradually declines over time with only partial recovery after backflushing [5,6]. Backflushing when a set pressure is recorded can improve the efficiency of the system. However, the interval between backflushes decreases over time due to the successive increases in the initial pressure after each backflush. Overall, the filtration time is significantly reduced [3].

The limited effectiveness of regular backflushes described above is believed to be due to fouling: particles enter the pores of the membrane, and are not readily removed by backflushes [7]. This is in contrast to caking - the build-up of particles on the surface of the membrane - which is effectively removed by backflushing. In this paper, we hypothesize that the underlying physical Preprint submitted to Journal of Membrane Science 


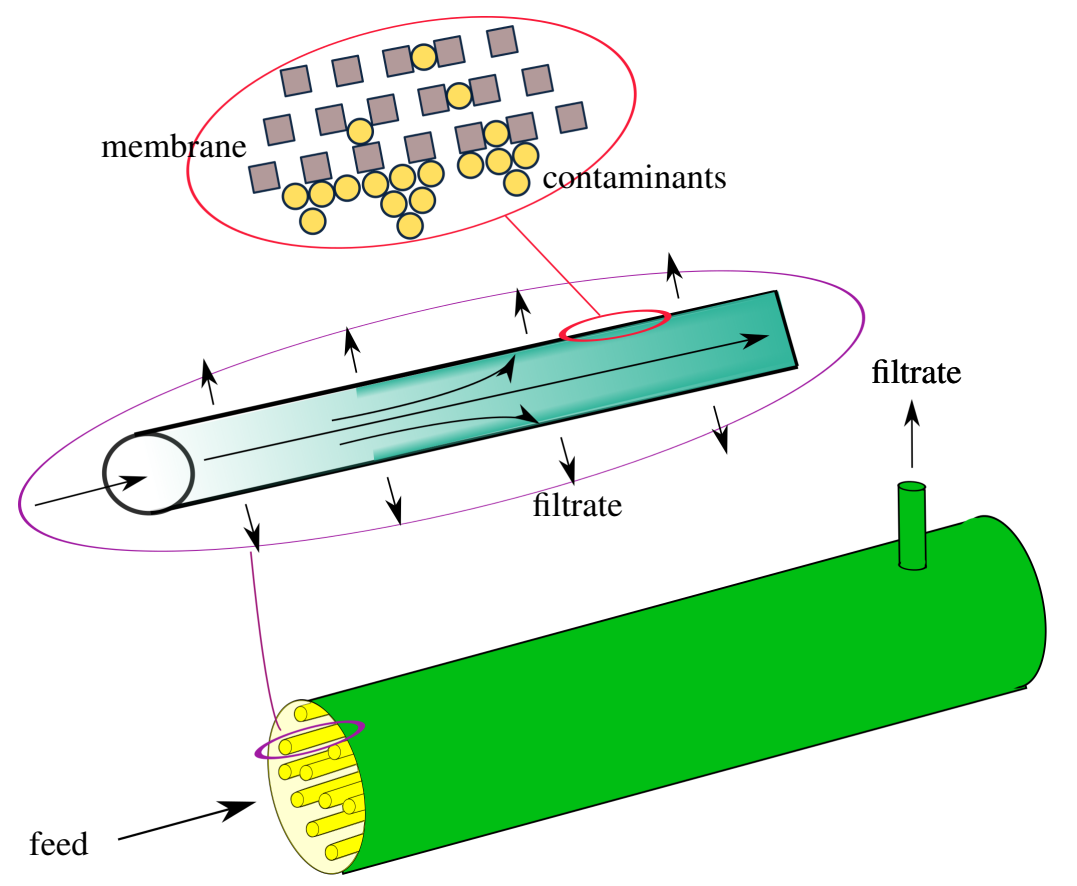

Figure 1: Sketch of a direct-flow membrane filtration device. A feed of contaminated water enters a tube with porous membrane walls. The membrane blocks the contaminants, allowing only clean water (filtrate) to pass through. 


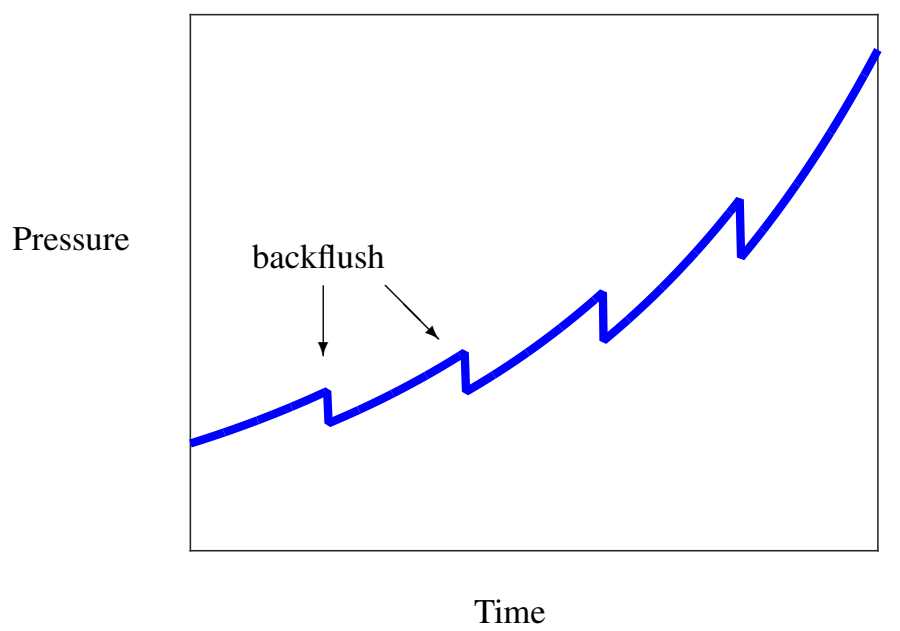

Figure 2: Sketch of the pressure versus time behaviour typically observed in a filtration device operating at constant flux. A cake layer of particles builds up on the membrane surface over time, increasing the pressure required to drive the constant flux. At regular intervals, backflushing is performed, removing the cake layer and reducing the filtration pressure. This causes the regular drops in pressure; note that, despite backflushing, the pressure never returns to its baseline value.

reason for this inability to remove fouling particles is the elastic deformation of the membrane during filtration and, in particular, the asymmetry in this deformation between forward flow (filtration) and backflushes. We model this deformation in detail, determining how it is coupled to the hydrodynamic pressures due to flow (which change because of caking and fouling). We also consider how deformation might be expected to modify the pore size, leading to increased fouling, and an accelerated decrease in the permeability of the membrane, as has been suggested qualitatively before [8]. All of these effects contribute to an increase in the driving pressure required for filtration.

Previous work has modelled fouling and caking extensively. In these models fouling is typically subcategorized as either concentration-polarization, complete, intermediate or internal blocking $[9,10,11,12,13]$. Here, we are concerned primarily with the interaction of fluid flow, fouling, and caking with the elastic response of the membrane and cake layer, and how these factors affect the progression of fouling and caking. For simplicity, we consider a model in which fouling only occurs via internal pore blocking: we assume that a single particle plugs a single pore, and any particle that reaches but does not enter a membrane pore instead forms part of a cake. We consider the case of microfiltration (see Appendix A) in which the osmotic pressure is small compared to the fluid pressure [14]. We may therefore neglect osmotic effects in our model. However, it is crucial that internal fouling reduces the membrane permeability.

The idea of elastic deformation resulting in increased fouling has previously been considered from a different perspective, the deformation of the contaminant itself, particularly under constant flux conditions [15]. For example, bacteria and macromolecules may deform to gain access to otherwise unavailable pores $[16,17]$. Similarly, in ultrafiltration, flexible polymers are stretched by the flow to the point that their projected cross-sectional area is smaller than the 
pore size, thus allowing the polymer to pass through the pore [18]. However, recent studies have demonstrated that the membrane itself may deform under high pressures and, further, that this deformation may lead to increased fouling as the pores increase in size [8].

The stress-strain relationship for hollow-fibre membranes has been characterized into three types: elastic strain, transitional strain, and plastic strain. The elastic strain region is typically valid for pressures of up to 10 bar [19], though there is considerable variation depending on the material and structure of an individual membrane. In any case, elastic strain is a factor for all types of filtration [20] while transitional and plastic strain may occur if the operating pressure is allowed to reach the highest values.

The deformation of a cake layer of soft colloids or deformable particles has also been studied. In particular, since the cake is observed to compress under pressure [21,22] while the membrane itself expands, there is an interesting coupling between membrane expansion and cake compression. It has been demonstrated that the attachment of particles to the cake layer, via van der Waals and electrostatic forces, is mostly irreversible [23]. We shall therefore assume that the cake layer is stable (not easily broken up) during filtration, as opposed to a backflush. (A novel route towards cake break-up is presented in [24].) Note, however, that growth of the cake layer increases the shear stress on its surface (by a combination of increased fluid velocity and reduced open area) and ultimately an equilibrium thickness is reached at which the shear stress is enough to overcome the adhesive forces. Microfiltration experiments report that this threshold cake thickness may be a significant fraction of the tube radius, up to $38 \%$ [25].

Finally, cake deposition and transmembrane pressure differences are often not uniform along the membrane: they usually increase and decrease, respectively, monotonically with distance along the membrane. However, in direct-flow systems (crossflow with a capped end) operation can be performed with a transmembrane pressure close to uniform [26]. To study the fundamental interaction of fouling and caking with elasticity in the simplest possible setting, we consider a 2D cross-section of a hollow-fibre membrane tube; we neglect axial variations.

In this paper, we present a model of the combination of caking, fouling, and elastic deformation described above. We begin by showing that the timescale of poroelastic response is much shorter than the rate of fouling. This motivates a quasi-static approximation to decouple the fouling and caking from the induced elastic responses: the elastic effects may be assumed to occur instantaneously, and appear as time-dependent parameters in a set of coupled ordinary differential equations (ODEs) that we derive for the fouling and caking. We then consider different operating strategies (particularly focussing on varying the rate of backflushing) that may be used to reduce fouling and the effects of elastic deformation.

\section{Mathematical Modelling}

\subsection{Setup}

The axisymmetric 2D setup that we consider is depicted schematically in Figure 3. The wall of the outer tube constitutes the porous membrane; we denote its elastic properties by $\hat{E}_{\mathrm{m}}$ and $v_{\mathrm{m}}$ (the Young's modulus and Poisson ratio, respectively). We use hats to denote dimensional quantities. Initially (i.e., before deformation), the membrane has internal radius $\hat{R}$ and thickness $\hat{d}_{\mathrm{m}}$. During filtration, a constant areal flux, $\hat{Q}$, of fluid flows radially from a source at the centre of the tube towards and through the membrane with a velocity $\hat{V}(\hat{r})$. During the backflush phase, the flux reverses sign so that the centre becomes a sink of strength $\hat{Q}$. We assume that the flow is quasi-steady and occurs at low Reynolds number, with a constant viscosity $\hat{\mu}$; we take the pressure outside the tube to be the (constant) pressure datum. 
We assume that the membrane consists of pores of equal size, $\hat{a}_{\text {pore }}$, that are uniformly distributed around the membrane. Due to the large number of pores typically found in a membrane, we describe the particle fouling and deposition via a continuum approach rather than modelling each pore individually. The pore size is allowed to vary during an experiment, $\hat{a}_{\text {pore }}=\hat{a}_{\text {pore }}(\hat{t})$, in response to the evolving deformation of the membrane (due to fouling and cake growth). The particles are assumed to have a distribution of sizes, $f(\hat{s})$, where $f(\hat{s})$ is non-zero only for $\hat{s} \in\left[\hat{a}_{\text {part }}^{\min }, \hat{a}_{\text {part }}^{\max }\right]$ : the minimum particle size is $\hat{a}_{\text {part }}^{\min }$, and the maximum particle size is $\hat{a}_{\text {part }}^{\max }$. (Different functional forms of $f$ may be considered, e.g. uniform, truncated Gaussian, etc. We take a uniform distribution to illustrate the phenomenology of the problem.) If $\hat{a}_{\text {pore }}(\hat{t})<\hat{a}_{\text {part }}^{\min }$ then particles are not able to foul the membrane and instead the cake layer grows; if $\hat{a}_{\text {pore }}(\hat{t})>\hat{a}_{\text {part }}^{\max }$ then all of the particles enter the membrane pores and contribute to fouling, but no cake layer grows. In general, particles in the size range $\left[\hat{a}_{\text {part }}^{\min }, \hat{a}_{\text {pore }}(\hat{t})\right]$ contribute to fouling, while particles in the size range $\left[\hat{a}_{\text {pore }}(\hat{t}), \hat{a}_{\text {part }}^{\max }\right]$ contribute to cake growth. The fraction of incoming particles that may enter the pore, $f_{p}$, is given by

$$
f_{p}(\hat{t})=\int_{\hat{a}_{\text {part }}^{\min }}^{\hat{a}_{\text {pore }}(\hat{t})} f(\hat{s}) \mathrm{d} \hat{s},
$$

with the remainder, the fraction $f_{c}=1-f_{p}$, contributing to cake growth. Finally, we shall assume that the membrane may be fouled even when a cake layer is present: particles in the cake may foul the membrane if the pores have become large enough for them to fit inside. For simplicity, and to highlight the fundamental aspect of this mechanism, we assume that backflushing removes the cake layer completely but does not alter the internal fouled state of the membrane. This could be easily modified to suit a different experimental observation.

Since filtration occurs at a constant flux, particles reach the membrane surface at a constant rate, $\lambda$, which is proportional to both the flow rate and particle concentration. The rules already given determine whether particles foul the membrane or form a cake layer. The growth of the cake layer adds resistance to the flow and exerts a mechanical stress on the membrane. We assume that this cake layer is also a poroelastic medium, with Young's modulus, $\hat{E}_{\mathrm{c}}$, and Poisson ratio, $v_{\mathrm{c}}$. As particles continue to be advected, the thickness of the cake, $\hat{d}_{\mathrm{c}}$, grows with time.

The domain $0<\hat{r}<\hat{R}+\hat{d}_{\mathrm{m}}$ divides into three regions: a fluid-filled space $\left(0<\hat{r}<\hat{R}-\hat{d}_{\mathrm{c}}\right)$, the cake $\left(\hat{R}-\hat{d}_{\mathrm{c}}<\hat{r}<\hat{R}\right)$, and the membrane itself $\left(\hat{R}<\hat{r}<\hat{R}+\hat{d}_{\mathrm{m}}\right)$. Our aim is to solve for the time evolution of the stresses with the cake layer and membrane, and to include the effect of membrane deflection on the fouling of membrane pores.

We use the parameter values given in Appendix A, unless otherwise stated.

\subsection{Poroelastic Timescale}

The poroelastic timescale, $\hat{T}_{\text {pe }}$, reflects the material's control over the storage and release of elastic energy: the diffusion and dissipation of fluid pressure. The timescale $\hat{T}_{\text {pe }}$ may be derived via a balance of the strain rate and elastic stress for the membrane [27],

$$
\hat{T}_{\mathrm{pe}}=\frac{\hat{\mu} \hat{R}^{2}}{\hat{E}_{\mathrm{m}} \hat{k}_{\mathrm{m}, 0}} .
$$

In water filtration, the viscosity is $\hat{\mu}=10^{-3} \mathrm{~Pa} \mathrm{~s}, \hat{R}=10^{-3} \mathrm{~m}$ is a typical internal tube radius, $\hat{E}_{\mathrm{m}}=10^{10} \mathrm{~Pa}$ is a typical membrane Young's modulus (see Appendix A), and $\hat{k}_{\mathrm{m}, 0}=10^{-16} \mathrm{~m}^{2}$ 


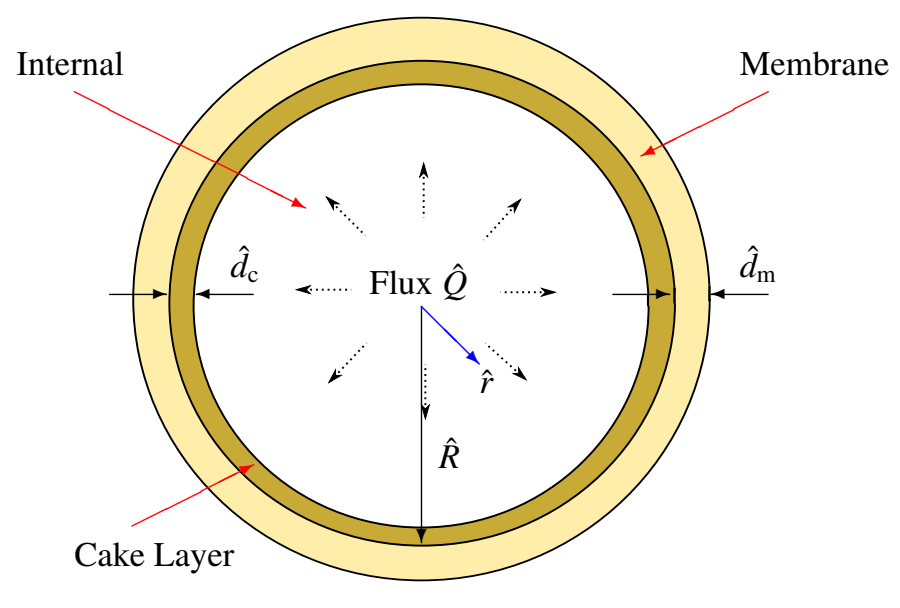

Figure 3: Cross-section through a cylindrical membrane. The filter has an undeformed internal radius $\hat{R}$. An areal fluid flux, $\hat{Q}$, flows radially from the centre of the tube with radial velocity $\hat{V}$. The membrane thickness is $\hat{d}_{\mathrm{m}}$ while the cake thickness, $\hat{d}_{\mathrm{c}}$, evolves as additional material is filtered.

is a typical membrane permeability. This gives a value of $\hat{T}_{\mathrm{pe}} \approx 1 \mathrm{~ms}$. The rates at which fouling and caking develop are on a timescale of the order of minutes (with backflushes performed approximately every 20 minutes). Hence, the poroelastic timescale is much shorter than the backflushing period, even for softer membranes. This justifies a quasi-static approximation, in which the elastic deformation is solved in steady state in response to the stresses for a particular cake size and amount of fouling.

\subsection{Fouling and Caking}

We present a simple model that accounts for the contribution of the incoming particles to both fouling and caking. We take the fraction of open, unfouled pores at a time $\hat{t}$ to be given by $\mathcal{F}(\hat{t})$. A particle only enters a pore if its size is at most that of the pore. We assume that any particle smaller than a pore is equally likely to occupy the pore. This means that the first sufficiently small particle to reach an open pore enters it and blocks it. (This is a simplification since the particle only has to be smaller than the pore to fit in and may not block it completely; further particles could then be added to the pore, provided that they fit in the space remaining, but we do not consider such complications here.) In this model, only a single layer of particles foul the membrane internally; we do not account for depth in the fouling, i.e., multiple particles fouling the same pore at different depths, but this could be incorporated using the ideas presented in [11].

If $\lambda \Delta \hat{t}$ particles, with size distribution $f(\hat{s})$, reach the membrane surface in an interval of time $\Delta \hat{t}$, the rate of fouling is given via the ODE,

$$
\frac{\mathrm{d} \mathcal{F}}{\mathrm{d} \hat{t}}=-\frac{\lambda}{N_{0}} f_{p}(\hat{t})
$$

where $f_{p}(\hat{t})$ is given in Eq. (1), and $N_{0}$ is the number of pores initially (which we expect to be the number of pores that can fit into the circumference, scaled with the porosity of the membrane, 
$\varphi_{\mathrm{m}}$, i.e. $\left.N_{0}=\varphi_{\mathrm{m}} \pi \hat{R} / \hat{a}_{\text {pore }}\right)$. Eq. (3) is to be solved with the initial condition,

$$
\mathcal{F}(0)=1,
$$

so that the membrane is initially unfouled.

The cake layer is an array of packed particles forming a porous medium with porosity $\varphi_{c}$, so that each additional monolayer of added particles leads to an increment $2 \bar{a}_{\text {part }} / \varphi_{c}$ in the cake thickness, $\hat{d}_{\mathrm{c}}$, where $\bar{a}_{\text {part }}$ denotes the mean particle size. For a membrane surface with circumference $2 \pi \hat{R}$, each layer of particles consists of $\varphi_{c} \pi \hat{R} / \bar{a}_{\text {part }}$ particles; that is, the perimeter of the surface divided by the diameter of a particle and scaled by the porosity of the cake layer. This of course is a first approximation; since the surface is curved, each layer has a smaller surface area, but such variations are insignificant if the cake thickness remains small compared to $\hat{R}$. The thickness of the cake layer, $\hat{d}_{\mathrm{c}}$, therefore grows according to

$$
\frac{\mathrm{d} \hat{d}_{\mathrm{c}}}{\mathrm{d} \hat{t}}=\frac{2 \lambda \bar{a}_{\text {part }}^{2}}{\varphi_{c}^{2} \pi \hat{R}}\left(1-f_{p}(\hat{t})\right) .
$$

We shall assume that, after each backflush, the cake layer is completely removed. If the backflushes are performed periodically at intervals of $\hat{t}_{\mathrm{bf}}$, then at the beginning of the $n^{\text {th }}$ filtration cycle, the initial condition on the cake layer is

$$
\hat{d}_{\mathrm{c}}\left(n \hat{t}_{\mathrm{bf}}\right)=0 .
$$

We non-dimensionalize the ODEs for fouling (3) and cake growth (5) by scaling the cake thickness with the undeformed membrane radius, $\hat{R}$, and the pore and particle sizes with the undeformed pore size, $\hat{a}_{\text {pore }}^{0}$. We consider the problem over the fouling timescale. Specifically, we let

$$
\hat{d}_{\mathrm{c}}=\hat{R} d_{\mathrm{c}}, \quad \hat{a}_{\text {pore }}=\hat{a}_{\text {pore }}^{0} a_{\text {pore }}, \quad \hat{a}_{\text {part }}=\hat{a}_{\text {pore }}^{0} a_{\text {part }}, \quad \hat{t}=\frac{\varphi_{\mathrm{m}} \pi \hat{R}}{\hat{a}_{\text {pore }} \lambda} t .
$$

The ODEs for fouling (3) and cake growth (5) may then be written as

$$
\begin{aligned}
\frac{\mathrm{d} \mathcal{F}}{\mathrm{d} t} & =-f_{p}(t), & \mathcal{F}(0) & =1, \\
\frac{\mathrm{d} d_{\mathrm{c}}}{\mathrm{d} t} & =\beta\left(1-f_{p}(t)\right), & d_{\mathrm{c}}\left(n t_{\mathrm{bf}}\right) & =0,
\end{aligned}
$$

where $f_{p}$ is given by Eq. (1) non-dimensionalized in the obvious way and

$$
\beta=\frac{2 \bar{a}_{\mathrm{part}}}{\varphi_{c} \hat{R}},
$$

represents the ratio of the average cake-layer depth of a monolayer to the membrane thickness. Typical parameters (provided in Appendix A) give the estimate $\beta \approx 0.02$.

\subsection{Poroelasticity}

In this section we consider the factors that determine the rate of fouling of the membrane. The distribution of particles in a given size range is given by the quantity $f_{p}(t)$ in Eq. (8), but 


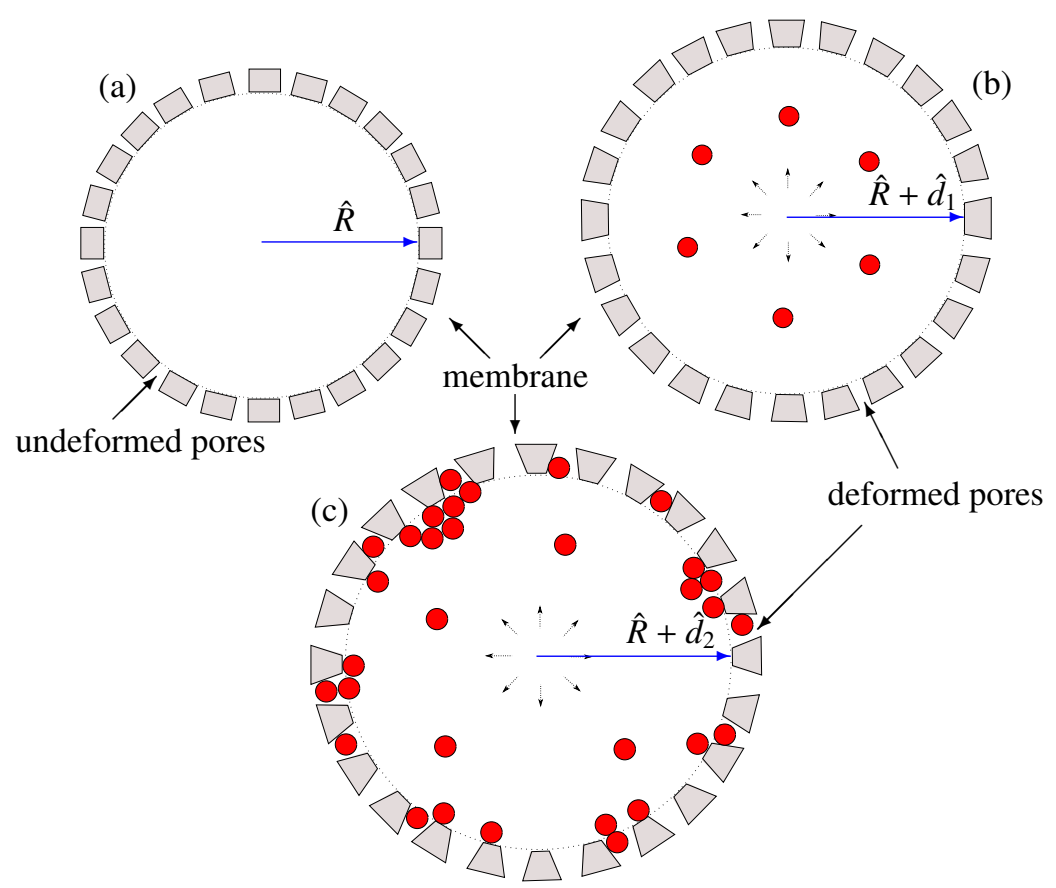

Figure 4: Schematic illustrating the expansion of a pore as fouling and caking occur. (a) The pores are initially uniform in size and undeformed, with radius $\hat{R}$. (b) A fluid flow through the membrane exerts a stress that deforms the membrane, inducing an increase in pore size. The tube radius increases by a small amount $\hat{d}_{1}$. (c) Fouling of the pores and the build up of a cake layer results in higher pressures and stresses, and hence further deformation of the membrane, by $\hat{d}_{2}>\hat{d}_{1}$, and to a larger pore size. The pressure required must increase to maintain a constant fluid flux through the membrane. The greater the fouling and thicker the cake layer, and hence the greater the deformation.

requires knowledge of the typical pore size at a particular operating pressure. We must therefore relate the fluid stress on the membrane to its deformation and thence to the change in the typical pore size. The deformation problem is made up of two parts: the deformation when no cake layer is present (at the beginning of the filtration process or after a backflush is performed) and when a cake layer is present. These two scenarios are fundamentally different.

Consider the schematic in Figure 4: in the absence of a cake layer (at the beginning of the experiment or immediately following a backflush) the membrane deforms elastically, by some distance $\hat{u}_{\mathrm{m}}^{0}=\hat{d}_{1}$, say, which is due solely to the fluid stress. When particles reach the membrane surface, if they are small enough they can enter and plug the membrane pores, reducing the permeability. As a result, a larger pressure must be applied to maintain a constant flux. Furthermore, if a cake layer builds up on the surface of the membrane, an additional mechanical stress is exerted on the membrane. Altogether, this increases the deformation, to $\hat{u}_{\mathrm{m}}=\hat{d}_{2}>\hat{d}_{1}$ say. In addition to this, the cake layer may be deformed by the flow. As the pore radius increases, $f_{p}(t)$ increases and so more irreversible fouling occurs. The fouling process worsens with each cycle.

\subsubsection{Elastic deformation}

Since the other aspects of the model are focused on deformations in the plane, we neglect axial elastic deformations and model the deformations of the membrane and cake via plane-strain 
linear elasticity. (We discuss the circumstances under which this might be expected to hold at the end of the paper.) We use the Navier-Cauchy equation with Terzaghi's principle to model the deformation of a porous material [27, 28]. The details for the poroelastic model are given in Appendix B. Here, we consider how the deformation affects the material properties of the membrane.

After deformation, the local dimensionless change in volume is given by the dilation

$$
\Delta:=\nabla \cdot \boldsymbol{u}=e_{r r}+e_{\theta \theta}=\frac{\mathrm{d} u}{\mathrm{~d} r}+\frac{u}{r},
$$

where $e_{r r}$ and $e_{\theta \theta}$ are the strain components, and $u=u(r)$ is the dimensionless radial deformation of the porous medium, with the deformation and radial coordinate both scaled with $\hat{R}$ [29]. Using the deformations given in Eq. (B.17), the local dilations (10) of the membrane and cake layer, $\Delta_{\mathrm{m}}(r)$ and $\Delta_{\mathrm{c}}(r)$ respectively, are given by

$$
\begin{array}{ll}
\Delta_{\mathrm{m}}(r)=-\frac{\Gamma}{2 \pi \Omega \mathcal{F}} \log r+2 A_{\mathrm{m}}, & 1<r<1+d_{\mathrm{m},}, \\
\Delta_{\mathrm{c}}(r)=-\frac{\Gamma \gamma}{2 \pi \Omega \omega \kappa} \log r+2 A_{\mathrm{c}}, & 1-d_{\mathrm{c}}<r<1,
\end{array}
$$

where $A_{\mathrm{m}}$ and $A_{\mathrm{c}}$ are (quasi-static) constants given in Eq. (B.18), which are determined as part of the solution to the problem, and $\Omega$ and $\Gamma$ are dimensionless parameters defined in (B.12a) and (B.12b). The dilation decreases with radial position $r$ (since the pressure decreases with $r$, the amount of compression/expansion changes too). An important observation for filtration applications is that, for typical parameter values discussed in Appendix $\mathrm{A}, \Delta_{\mathrm{m}}>0$ (the membrane expands) while $\Delta_{\mathrm{c}}<0$ (the cake is compressed).

\subsubsection{Changes in pore size and porosity}

While the mixture of void space and solid may change its volume locally, as discussed above, we assume that the solid portions of the porous media are both incompressible: any local dilation is accommodated solely by a local change in porosity. With this assumption, the deformed porosity, $\varphi_{\mathrm{d}}$, can be written in terms of the undeformed (uniform) porosity, $\varphi_{0}$, as

$$
\varphi_{\mathrm{d}}=1-\frac{1-\varphi_{0}}{1+\Delta} \approx \varphi_{0}+\Delta\left(1-\varphi_{0}\right)
$$

for small deformations, $\Delta \ll 1$, consistent with our assumption of linear elasticity. Here $\Delta$ is given in Eq. (11) for the membrane and cake layer. However, we emphasize that, in general, $\Delta=\Delta(r)$, so that the initially uniform porosity becomes inhomogeneous, because of the inhomogeneity of the deformations. It is also possible that the permeability becomes anisotropic as a result of this deformation, but we neglect such possibilities here.

We can now quantify the change in pore size. Suppose that, in the undeformed reference state, either porous media is composed of $\hat{M}$ pores per unit area, each with equal size $\hat{a}_{\text {pore }}^{0}$. The porosity, $\varphi_{0}$, is the volume fraction of the pores, $\varphi_{0}=\hat{M} \pi\left(\hat{a}_{\text {pore }}^{0}\right)^{2}$. After deformation, the pores have a new radius, $\hat{a}_{\text {pore }}$. However, the number of pores is unchanged following deformation and so the new pore size may be determined using the deformed porosity Eq. (12), with $\varphi_{\mathrm{d}}=\hat{M} \pi \hat{a}_{\text {pore }}^{2}$, to give, in dimensionless terms,

$$
a_{\text {pore }}(t) \approx 1+\frac{1}{2} \Delta \frac{1-\varphi_{0}}{\varphi_{0}}
$$


where we have assumed $\Delta \ll 1$. As expected, the pore size increases for $\Delta>0$ and decreases for $\Delta<0$ (recalling that $0<\varphi_{0}<1$ ).

To understand fouling, the most important pore size is that at the membrane surface, since this determines whether a particle is accepted or rejected by the membrane. Using the displacement field within the membrane, (11a), to calculate $\Delta=\Delta_{\mathrm{m}}(r=1)$, we find that the pore size (13), $a_{\text {pore }}(t)$, takes the form

$$
a_{\mathrm{pore}}(t)=1+A_{\mathrm{m}}(t) \frac{1-\varphi_{\mathrm{m}, 0}}{\varphi_{\mathrm{m}, 0}} .
$$

Here the constant, $A_{\mathrm{m}}=A_{\mathrm{m}}(t)$, is determined from the quasi-static membrane deformation solution (B.18), and is now a time-dependent parameter that depends on the parameters of the system, including the dimensionless cake thickness, $d_{\mathrm{c}}(t)$, and the degree of fouling, $\mathcal{F}(t)$.

At this stage, we have assumed that the deformation of the membrane is known. However, in reality this deformation depends on the fluid pressure, which in turn depends on how difficult it is to pump the imposed flux $\hat{Q}$ through the membrane or, in other words, the permeability of the porous media. We therefore turn to determining this next.

\subsubsection{Permeability of the porous media}

A common model of porous media relates the permeability, $\hat{k}$, of a porous medium to its porosity, $\varphi$, and pore size, $\hat{a}_{\text {pore }}$. There are many choices for this constitutive relation, but a popular choice is the Kozeny-Carman relation [28, 30]. Since both $\varphi$ and $\hat{a}_{\text {pore }}$ depend on the dilation we may write

$$
\hat{k}_{\mathrm{d}}=\frac{\varphi_{\mathrm{d}}^{3}}{\left(1-\varphi_{\mathrm{d}}\right)^{2}} \frac{\hat{a}_{d}^{2}}{36 \tau} \approx \hat{k}_{0}\left(1+k_{1} \Delta(r)\right)
$$

where we have used Eqns. (12) and (13), and neglected $O\left(\Delta^{2}\right)$ terms. In (15),

$$
\begin{aligned}
& \hat{k}_{0}=\frac{\varphi_{0}^{3}}{\left(1-\varphi_{0}\right)^{2}} \frac{\hat{a}_{0}^{2}}{36 \tau}, \\
& k_{1}=\frac{2\left(2-\varphi_{0}\right)}{\varphi_{0}},
\end{aligned}
$$

where $\tau$ is the tortuosity of the pore space, which is assumed to be constant.

While Eq. (15) gives the permeability of those pores that are unblocked, we must recall that some portion, $1-\mathcal{F}$, of the pores are fouled, and so the effective membrane permeability is timedependent, $\hat{k}_{\mathrm{m}, 0} \mathcal{F}(t)$. We non-dimensionalize the permeabilities by scaling with the leading-order unfouled membrane permeability $\hat{k}_{\mathrm{m}, 0}$ so that

$$
\begin{aligned}
k_{\mathrm{m}} & =\mathcal{F}\left(1+k_{\mathrm{m}, 1} \Delta_{\mathrm{m}}\right), \\
k_{\mathrm{c}} & =\kappa\left(1+k_{\mathrm{c}, 1} \Delta_{\mathrm{c}}\right),
\end{aligned}
$$

where $\Delta_{\mathrm{m}}$ and $\Delta_{\mathrm{c}}$ are the membrane and cake-layer dilations, given in eqn (11), $\kappa$ is the ratio of the cake to membrane permeabilities (B.9), and $k_{\mathrm{m}, 1}$ and $k_{\mathrm{c}, 1}$ are the relevant $k_{1}(16 \mathrm{~b})$ for the membrane and cake, respectively.

We have considered the undeformed membranes as a porous medium with uniformly distributed, identical pores. However, the elastic deformation is dependent on the radial position, $r$, 
as shown by the expressions for the dilation $\Delta_{\mathrm{m}}$, (11a). As a result, the porosity (12), pore size (13), and permeability (17a) all depend on $r$. In this sense, the membrane has been transformed into an asymmetric membrane in which the pores are larger at the inner surface (where rejection is meant to occur) than they are further into the membrane.

The effect of fouling on the the membrane permeability is two-fold. Firstly, from (17a) we see that permeability decreases with fouling, as would be expected. However, since fouling is also expected to increase the driving pressure and hence the elastic deformation, we find that there are higher order effects of fouling on permeability via the dilation $\Delta_{\mathrm{m}}$, which depends on pressure. In principle, this dilation can increase or decrease the permeability (depending on the sign of $\left.\Delta_{\mathrm{m}}\right)$.

\subsubsection{Coupling to the fluid flow}

The permeability of the membrane has a direct impact on the pressure gradient across the medium required to drive the (fixed) flux, as expressed by Darcy's law (B.2). As a result, the elastic expansion of the membrane has two contrasting effects on the flow: firstly, there may be an increase in fouling (caused by an increase in pore size as discussed in the previous subsection). Secondly, however, the elasticity-induced increase in pore size, and the concomitant increase in permeability, may reduce the pressure required to drive filtration.

Modifying the standard Darcy equation (B.2) to account for the variable permeability of (17a) we find that the dimensionless Darcy velocity through the membrane, scaled with $\hat{Q} / \hat{R}$, is

$$
V=-\left(1+\Delta_{\mathrm{m}}(r) k_{\mathrm{m}, 1}\right) \mathcal{F} \frac{\mathrm{d} p}{\mathrm{~d} r}
$$

where $k_{\mathrm{m}, 1}(16 \mathrm{~b})$ is a constant specified for the membrane in (17a), $\Delta_{\mathrm{m}}=\Delta_{\mathrm{m}}(r)$ is given by (11a) and we have scaled pressure via $\hat{p}=\left(\hat{Q} \hat{\mu} / \hat{k}_{\mathrm{m}}\right) p$. Substituting the velocity profile, $V=1 /(2 \pi r)$ from (B.8a) and rearranging we find that

$$
\frac{\mathrm{d} p}{\mathrm{~d} r}=-\frac{1}{2 \pi \mathcal{F}\left(1+\Delta_{\mathrm{m}}(r) k_{\mathrm{m}, 1}\right) r} \approx-\frac{1}{2 \pi \mathcal{F} r}+\frac{A_{\mathrm{m}} k_{\mathrm{m}, 1}}{\pi \mathcal{F} r}-\frac{\Gamma k_{\mathrm{m}, 1} \log r}{4 \pi^{2} \Omega \mathcal{F}^{2} r},
$$

for $1<r<1+d_{\mathrm{m}}$, with $A_{\mathrm{m}}, \Gamma, \Omega$, and $k_{\mathrm{m}, 1}$ all constants that are defined in Appendix B; furthermore, we have exploited the small-strain approximation $\Delta_{\mathrm{m}}(r) \ll 1$.

Integrating (19) subject to the ambient pressure condition, $p\left(r=1+d_{\mathrm{m}}\right)=0$, we find that the pressure field in the membrane (including the elastic correction), $p_{m}$, is

$$
p_{\mathrm{m}}=\frac{1}{2 \pi \mathcal{F}} \log \left(\frac{1+d_{\mathrm{m}}}{r}\right)+\frac{k_{\mathrm{m}, 1}}{\pi \mathcal{F}} \log \left(\frac{1+d_{\mathrm{m}}}{r}\right)\left[\frac{\Gamma}{8 \pi \Omega \mathcal{F}} \log \left[r\left(1+d_{\mathrm{m}}\right)\right]-A_{\mathrm{m}}\right],
$$

for $1<r<1+d_{\mathrm{m}}$. Here, the first term reproduces the pressure in the membrane without elastic deformation (B.8b). Examining the second term on the RHS of (20), we note that pore blocking increases the pressure change across the membrane (last term) whereas elasticity decreases this (second term). This reflects that the pressure needed to drive the flow in the flexible case is less than that in the rigid, undeformed case, because of the expansion of the pores.

We may repeat the above steps to determine the effect of the (compression-induced) permeability of the cake. We find now that

$$
\frac{\mathrm{d} p}{\mathrm{~d} r}=-\frac{1}{2 \pi \kappa\left(1+\Delta_{\mathrm{c}}(r) k_{\mathrm{c}, 1}\right) r} \approx-\frac{1}{2 \pi \kappa r}+\frac{A_{\mathrm{c}} k_{\mathrm{c}, 1}}{\pi \kappa r}-\frac{\Gamma \gamma k_{\mathrm{c}, 1} \log r}{4 \pi^{2} \Omega \omega \kappa^{2} r},
$$




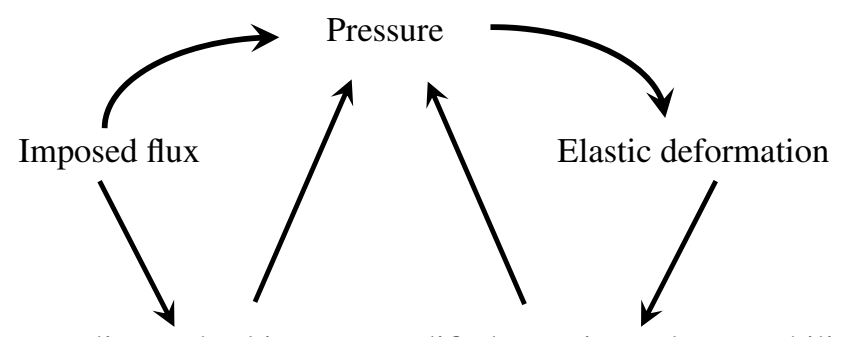

Fouling and caking Modified porosity and permeability

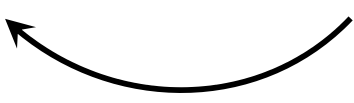

Figure 5: Flow chart illustrating the fouling process and our solution methodology.

with $A_{\mathrm{c}}, \Gamma, \Omega, \gamma, \omega, \kappa$ and $k_{\mathrm{c}, 1}$ all constants defined in Appendix B; again $\Delta_{\mathrm{c}}(r) \ll 1$ for consistency with our small deformation theory.

Again (21) may be integrated (subject to the condition that the pressure be continuous at the membrane-cake interface) to give an elastically corrected pressure field within the cake layer, say $p_{c}$, given by

$$
\begin{aligned}
p_{\mathrm{c}}= & \frac{1}{2 \pi \mathcal{F}} \log \left(1+d_{\mathrm{m}}\right)-\frac{1}{2 \pi \kappa} \log r \\
& +\frac{k_{\mathrm{m}, 1}}{\pi \mathcal{F}} \log \left(1+d_{\mathrm{m}}\right)\left[\frac{\Gamma}{8 \pi \Omega \mathcal{F}} \log \left(1+d_{\mathrm{m}}\right)-A_{\mathrm{m}}\right]-\frac{k_{\mathrm{c}, 1}}{\pi \kappa} \log r\left[\frac{\Gamma \gamma}{8 \pi \Omega \omega \kappa} \log r-A_{\mathrm{c}}\right],
\end{aligned}
$$

valid for $1-d_{\mathrm{c}}<r<1$. Here, the first term corresponds to the leading-order pressure required, accounting for the cake layer, in the absence of deformation, as given by (B.8b). Examining the right-hand side of (22), we see that any blocking of the membrane is felt in the pressure across the cake layer as expected. However, here elasticity increases the pressure change across the cake layer as it is compressed. In figure 5 we illustrate the steps taken in the form of a flow chart.

The quantity that is of most interest from a practical point of view is the driving pressure, $\hat{P}_{\text {drive }}$; this is the constant pressure within the internal void region and is given by evaluating the pressure field (22) at the inner cake boundary $\left(r=1-d_{\mathrm{c}}\right)$. We find that

$$
\begin{aligned}
\hat{P}_{\text {drive }}= & \frac{1}{2 \pi \mathcal{F}} \log \left(1+d_{\mathrm{m}}\right)-\frac{1}{2 \pi \kappa} \log \left(1-d_{\mathrm{c}}\right) \\
& +\frac{k_{\mathrm{m}, 1}}{\pi \mathcal{F}} \log \left(1+d_{\mathrm{m}}\right)\left[\frac{\Gamma}{8 \pi \Omega \mathcal{F}} \log \left(1+d_{\mathrm{m}}\right)-A_{\mathrm{m}}\right] \\
& -\frac{k_{\mathrm{c}, 1}}{\pi \kappa} \log \left(1-d_{\mathrm{c}}\right)\left[\frac{\Gamma \gamma}{8 \pi \Omega \omega \kappa} \log \left(1-d_{\mathrm{c}}\right)-A_{\mathrm{c}}\right] .
\end{aligned}
$$

Before moving on to discuss our results for the (quasi-static) evolution of filtration and fouling, it is worthwhile studying the form for the driving pressure (23). Fouling, characterized by 
$\mathcal{F}$, and caking, characterized by the cake thickness $d_{\mathrm{c}}$, appear explicitly in (23) and that both act to increase the driving pressure (since $\mathcal{F}<1$ and $\delta_{c}>0$ ), as should be expected. We also note that the first two terms of (23) represent the leading-order pressure without any deformation while the third and fourth terms represent the first-order corrections to the pressure due to the expansion of the membrane (which reduces the driving pressure) and compression of the cake (which increases the driving pressure), respectively.

The approximations (20), (22) and (23) hold for small deformations, $\Delta_{\mathrm{m}}(r) \ll 1$ and $\Delta_{\mathrm{c}}(r) \ll 1$. As deformations get larger, the driving pressure $\hat{P}_{\text {drive }}$ must be determined by direct numerical integration of (19) and (21).

Finally, we note that the effect of elastic deformation on the porosity, pore size, and permeability enter as first-order changes to the fluid flow and membrane fouling. Other physical parameters, such as the Young's modulus $\hat{E}$ and Poisson ratio $v$ may well be affected by elastic deformation. However, since these changes in parameters would alter the fluid flow and membrane fouling at still higher order, we neglect such variations here.

\section{Results}

The driving pressure given in (23) illustrates the detrimental effect fouling can have on the efficiency of the system. As fouling $($ i.e., decreasing $\mathcal{F}$ ) reduces the permeability of the membrane, the driving pressure must increase to maintain a constant flux. Eventually the driving pressure becomes so high that the membrane is no longer practical.

In this section we present results for how the fouling and cake growth develop as functions of time. This is achieved by solving the set of ODEs (8) numerically. However, a key indicator of how fouling and cake growth would affect filtration is through the evolution of the driving pressure given by (23).

A key concern is on the effect of regular backflushing and so we envisage performing a backflush at regular intervals of time $t_{\mathrm{bf}}$; we assume that each backflush fully removes the cake layer, which is expressed via the condition (8b). The system (8) is solved numerically, with a particle size distribution, $f$, and the membrane surface pore size, $a_{\text {pore }}(t)$, given by Eq. (14). The numerical integration is performed using the MATLAB routine ode45. At each time step, $\Delta t$, the deformed pore size, $a_{\text {pore }}(t)$ (14), is updated for the current values of $\mathcal{F}(t)$ and $d_{\mathrm{c}}(t)$ that determine its elastic deformation. As $t$ passes through $n t_{\mathrm{bf}}, d_{\mathrm{c}}$ is reset to 0 (representing a backflush), but $\mathcal{F}(t)$ remains unchanged. This is outlined in Algorithm 1.

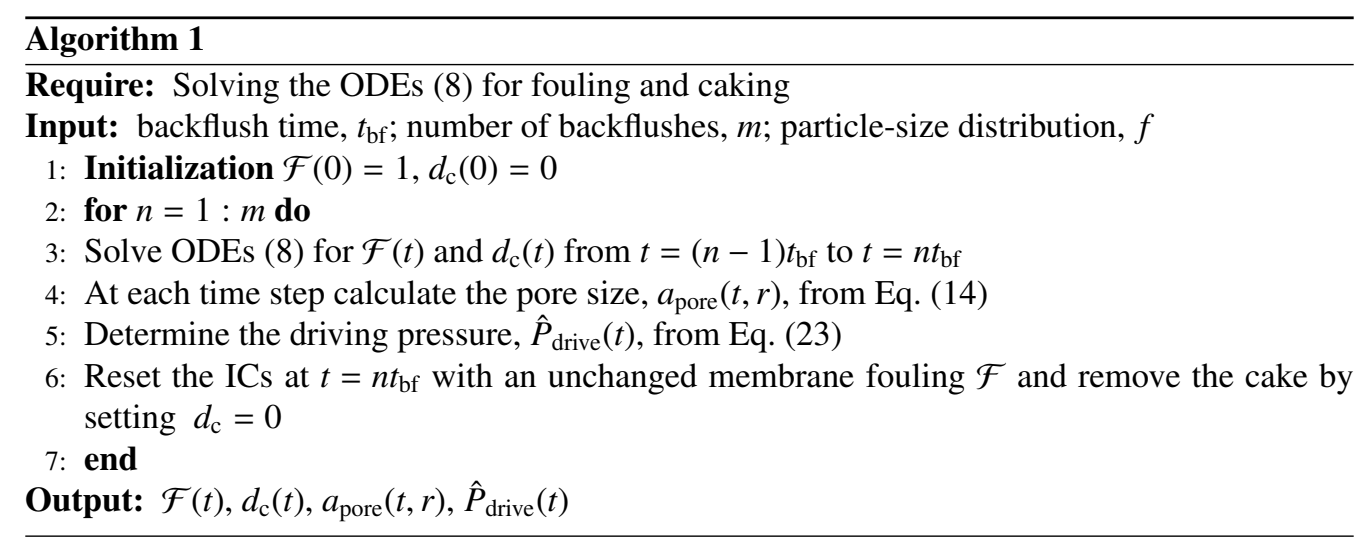




\subsection{Development of fouling and caking}

To illustrate the range of behaviours exhibited by our model, we first assume that the particle distribution, $f$, is uniform with a minimum particle size $\hat{a}_{\text {part }}^{\min }=4 \mu \mathrm{m}$ (microfiltration) and maximum particle size $\hat{a}_{\text {part }}^{\max }=5 \mu \mathrm{m}$. The undeformed pore size is taken to be $4.1 \mu \mathrm{m}$ so that initially $10 \%$ of the particles are able to foul the membrane.

In figure 6 we show how the various properties of the system evolve with time, depending on the number of backflushes (zero, one or two). For later comparison, the dashed curves in figure 6 show how fouling and cake growth proceed when elastic effects are neglected. In the undeformed case, the pore size, $a_{\text {pore }}$, remains constant and the rate of fouling and cake buildup are now decoupled. The right-hand side of each ODE is constant and, hence, the system has solutions that are linear with time. The fouling follows a linear progression that remains unchanged with the number of backflushes (Figure 6(b)), whereas the cake layer grows linearly until a backflush removes it and cake growth starts over again (Figure 6(c)).

Adding in the various effects of elasticity discussed above gives rise to the solid curves shown in figure 6. The key difference here is that initially the rate of fouling is relatively weak (since only $10 \%$ of particles are small enough to foul the membrane, as in the rigid case). However, as the cake grows, so does the pressure, which in turn opens the pores (Figure 6(a)), allowing more particles to foul the membrane. The effect of this can be seen in the proportion of the membrane that is fouled, which decreases significantly faster in the elastic case than in the rigid case (Figure 6(b)). The increased fouling also slows the rate of cake growth (Figure 6(c)) since particles enter the system at the same rate and more are entering the pore spaces. Note also that these effects grow more noticeable with time: the rate of fouling increases with time, as has been widely acknowledged in the membrane industry [6].

The number of backflushes also alters the elastic response of the system. Elastic deformation leads to additional fouling and so the pore size after each subsequent backflush remains larger than at the beginning of the last cycle. Nevertheless, it is primarily the size of the cake layer that causes the pores to enlarge: each time the cake layer is removed by backflushing, the pore size drops (Figure 6(a)). As a result, more regular backflushes reduce the number of particles that can foul the membrane and reduces the rate of fouling (Figure 6(b)), as expected.

As the filtration process generally operates at constant flux, its efficiency is measured by the driving pressure, $\hat{P}_{\text {drive }}$, which responds quasi-statically to changes in $\mathcal{F}$ and $d_{\text {c }}$. In Figure $6(\mathrm{~d})$ we show the evolution of the driving pressure for the system. This figure resembles the schematic of the driving pressure in a typical membrane filtration operation (Figure 2) used to motivate this study. Varying the parameters in our model would change the quantitative nature of the graph while leaving the qualitative shape unaffected; this would allow us, in principle, to compare with experimental data.

Finally, if the pore size is initially smaller than all the particles, then no fouling occurs until the fluid and cake stresses deform the membrane sufficiently that particles can foul the membrane. The system then behaves as described above. We will discuss this, in the context of a strategy to prevent fouling, in $\S 3.3$.

\subsection{Competing mechanisms}

The preliminary numerical analysis of the preceding section shows that the effects of elasticity are not straightforward: the elasticity of the membrane increases the pore size, and hence the permeability of the membrane, decreasing the driving pressure. However, this increase in pore size also means that a greater proportion of particles are able to enter the pores and foul the membrane irreversibly, which ultimately increases the pressure. There is thus a competition between 
(a)

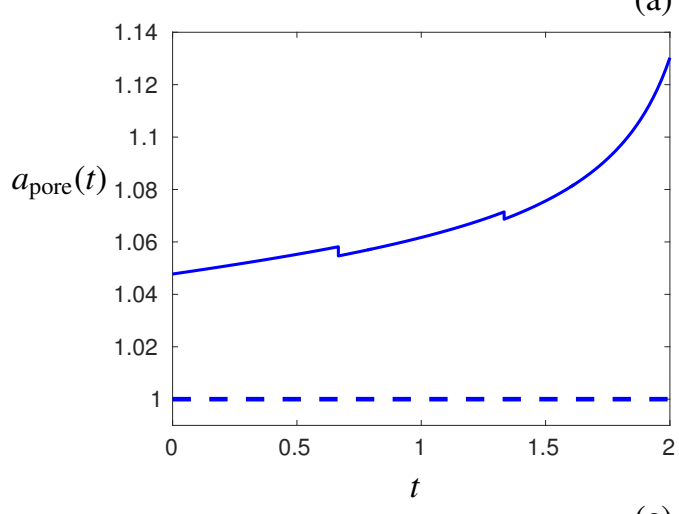

(c)

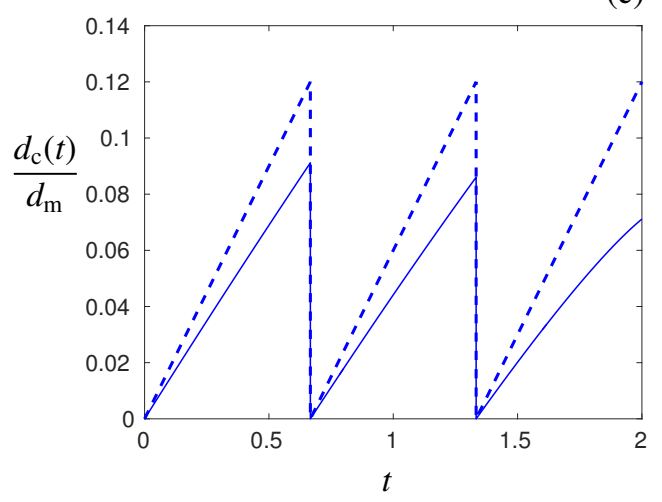

(b)

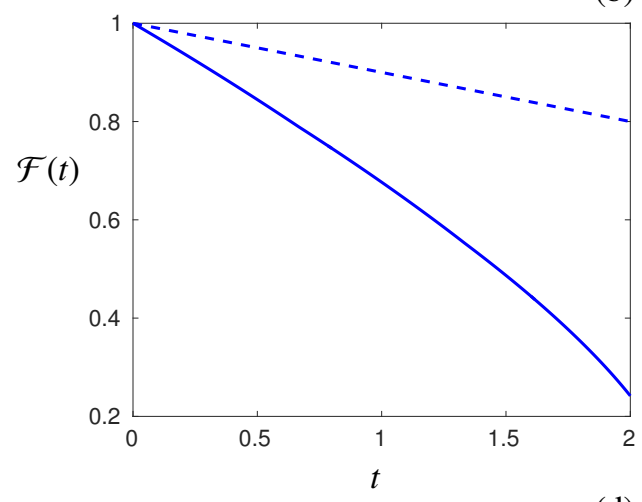

(d)

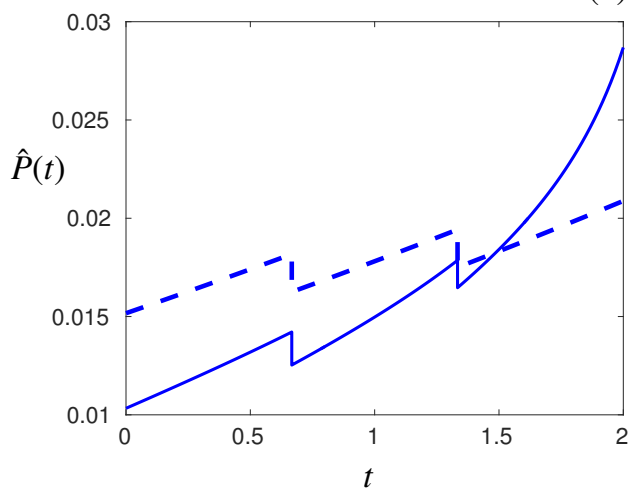

Figure 6: The evolution of (a) pore size, (b) proportion of unfouled pores, (c) cake thickness (as a fraction of membrane thickness) and (d) driving pressure, $\hat{P}$ (23), with time. Results are shown for a deformed membrane (solid curves) and the rigid case (dotted curves). Here, two backflushes are performed during operation and the dimensionless membrane thickness is $d_{\mathrm{m}}=0.1$. 

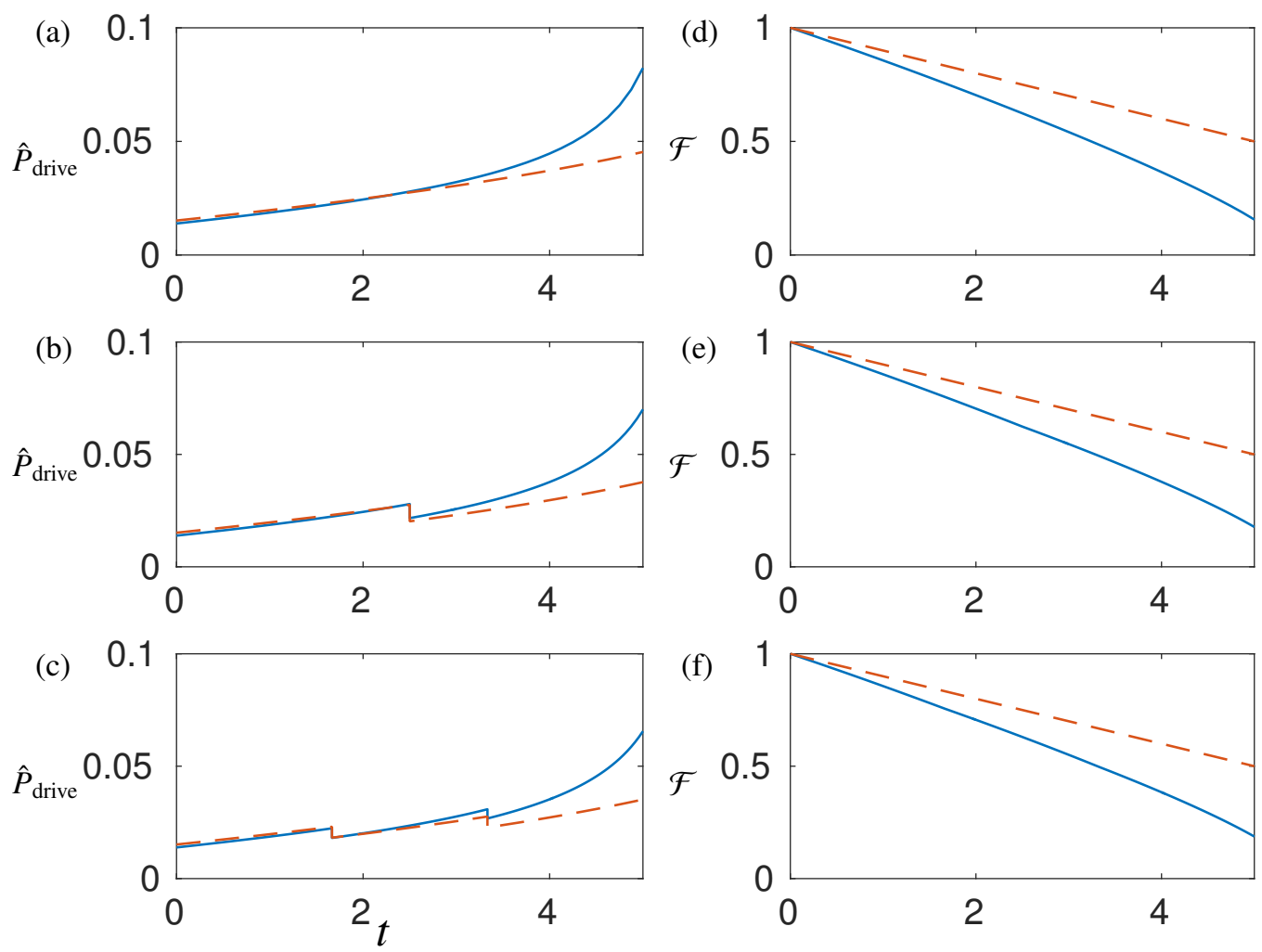

Figure 7: Evolution of driving pressure $\hat{P}_{\text {drive }}(t)(23)$ and fouling $\mathcal{F}(t)(8 \mathrm{a})$ over a time period [0,3] with (a,d) no backflushes, (b,e) one backflush, and (c,f) two backflushes. We compare the pressure with (solid) and without (dashed) elastic effects. Initially, elastic effects reduce the driving pressure, but increased fouling means that after some time the pressure exceeds that of the rigid case.

increased permeability and increased fouling as a result of elastic deformation. An analogous, albeit reversed, competition occurs in the cake layer: elastic deformation squeezes the cake layer, reducing its permeability but, because of the fouling of the membrane caused by deformation, the rate of cake growth is slowed (see Figure 6(c)).

To see the effect of these competing mechanisms, in figure 7 we illustrate the decrease in driving pressure (left panel) and fouling (right panel) over time as we increase backflushing rates, with (solid) and without (dashed) elastic effects. Initially, elastic effects reduce the driving pressure by opening the pores to allow fluid to pass more easily. However, eventually this is detrimental to the system as increased fouling clogs up the pores. Then, the pressure becomes significantly greater with elastic effects than without.

Driving pressures overall are reduced as the number of backflushes that are performed in a given interval of time increases. Figure 8 shows how the amount of fouling and driving pressure change after a fixed time interval of filtration, $t_{f}=5$, but with different numbers of backflushes performed in that interval. The results of figure 8 are therefore plotted as a percentage reduction in fouling (a) and driving pressure (b) compared with the case of no backflushing. Note that for increasing frequency of backflushing, the degree of fouling and driving pressure at the end 

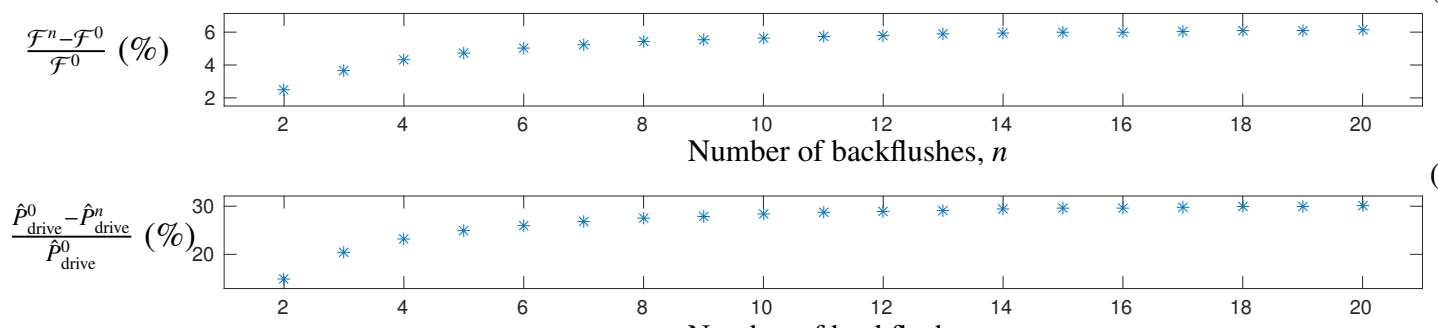

Number of backflushes, $n$
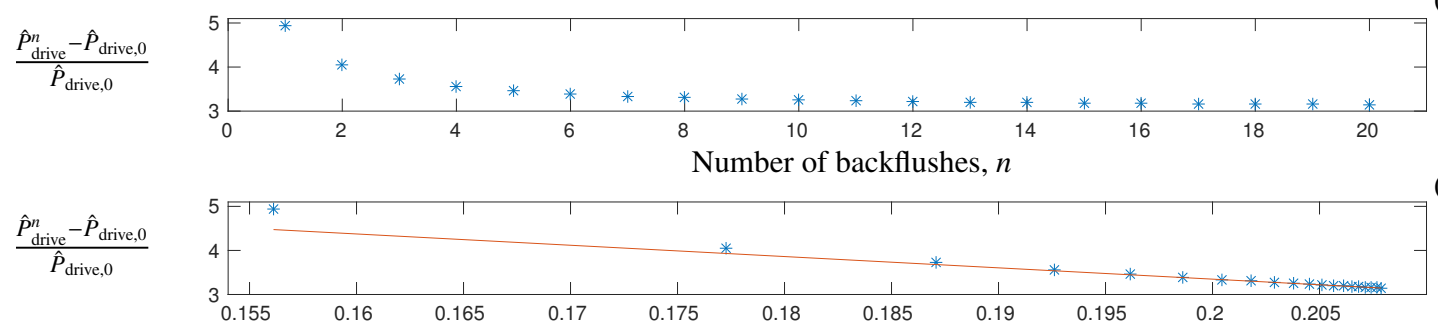

Fouling, $\mathcal{F}$

Figure 8: The effects of backflushing on pressure and fouling for a number of backflushes $n$ performed in a fixed time interval. (a) The percentage reduction in fouling (8a). (b) The percentage decrease in relative driving pressure (23) at the final time $\hat{t}_{f}$. As the number of backflushes performed in a fixed time interval increases, both the fouling and driving pressure are reduced compared with the case of no backflushing. (c) The pressure change between start and end times (initially, $\hat{P}_{\text {drive }, 0}=\hat{P}_{\text {drive }}(t=0)$ ). (d) The pressure change between start and end times as a function of the final amount of fouling for different numbers of backflushes (cf. (c)). 
of the numerical experiment decrease, but that ultimately the effect plateaus after approximately 10 backflushes in the cycle (figure 8). This plateau in the percentage reduction in fouling and driving pressures reaches $6 \%$ and $30 \%$, respectively. The pressure increase during each run is reduced significantly by backflushing, from a factor of approximately 5 to 3 (Figure 8(c)), with a direct correspondence to a reduction in fouling. The backflushing effect on pressure and fouling shows an approximately linear relationship between the two (d).

For long enough operating times, pressure always increases regardless of the material parameters, and eventually with fouling can become excessively large (Figure 6(d)). However, we can consider operating conditions to prevent this: not only to remain in a region where elasticity reduces the pressure, but also one in which the pores do not expand enough to allow any fouling.

\subsection{A strategy for fouling prevention}

Pore fouling tends to be irreversible, and has more severe consequences than cake buildup (which can be mitigated by backflushing). However, internal fouling cannot occur if the pore size is maintained at a value below that of the minimum particle size. Our previous results have shown that a significant contributor to the opening of pores that can be important in fouling is the mechanical stress of the cake layer acting on the membrane. It is natural then to wonder whether one can perform backflushes often enough that this mechanical pressure does not increase to the point at which the pores are opened enough to allow fouling to occur. In this section, we show that it is, at least in principle, possible to operate filtration in such a regime.

Suppose the smallest particle is a fraction $\eta$ larger than the undeformed (and uniform) pore size. Fouling will only be possible at times $t>t_{\text {crit }}$ where $t_{\text {crit }}$ is defined by the equation

$$
\eta=a_{\text {pore }}\left(t_{\text {crit }}\right)-1=A_{\mathrm{m}}\left(t_{\text {crit }}\right)\left(\frac{1}{\varphi_{0}}-1\right) .
$$

Here we have used (14) and $A_{\mathrm{m}}$ defined in (B.18), which depends on the cake thickness $d_{\mathrm{c}}$ (figure 9(a)). In the absence of fouling, the fraction of active membrane pores is $\mathcal{F}=1$ for all times. The cake-growth ODE (8b) can then be solved independently. The time, $t_{\text {crit }}$, at which the value of $d_{\mathrm{c}}$ is large enough to extend the pores by a fraction $\eta$ determines the minimum backflush frequency, $f_{\mathrm{bf}}^{*}=1 / t_{\text {crit }}$.

A proof of concept of this strategy is shown in Figure 9(b); again we note that the procedure must be tuned for other material and operating parameters. In dimensional terms we take pores of initial diameter $3.957 \mu \mathrm{m}$, with the particle size distribution being uniform on $4 \mu \mathrm{m} \leq a_{0} \leq 5 \mu \mathrm{m}$. This corresponds to $\eta=0.00525$. The cake thickness required to achieve fouling is then $d_{\mathrm{c}}=0.1096 d_{\mathrm{m}}$, via (24). Prior to this point, the solution to (8b) is linear in time; in particular, we find that in half a time unit the cake grows by an amount $\Delta d_{\mathrm{c}}=0.1 d_{\mathrm{m}}$. Hence, operating at a backflush frequency of 2 per unit time should be just enough to prevent fouling. We demonstrate this by backflushing at two different frequencies: once and twice per unit time over 350 time units (we consider such a long time interval to demonstrate the detrimental effect of fouling once it begins to occur). The inset of Figure 9(b) illustrates the onset of fouling in each cycle once the cake layer has had enough time to grow.

\section{Discussion \& Conclusions}

In this paper, we have developed a model for the evolution of fouling and cake development in membrane filtration. Our model incorporates the effect of a small elastic (plane-strain) deformation of the membrane and cake layer; the two phenomena of fouling and caking are coupled by 

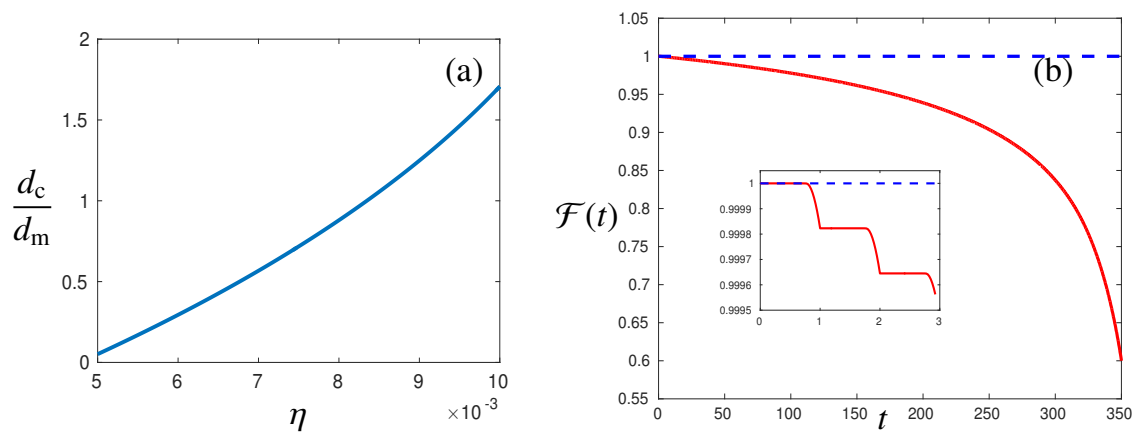

Figure 9: A proof of concept for a strategy to prevent fouling. We consider a uniform distribution of particles in the range $4 \mu \mathrm{m} \leq a_{0} \leq 5 \mu \mathrm{m}$, and a membrane pore size $3.957 \mu \mathrm{m}$. Once the membrane pores expand beyond $4 \mu \mathrm{m}$, fouling begins to occur. (a) The critical cake thickness, $d_{\mathrm{c}}$, at which the pore size increases enough to allow some fouling as a function of the relative difference in radii, $\eta$, between the smallest particle and the undeformed pore size. Provided that the critical pore size remains below this value, then (according to our model) fouling will not occur. (b) The time evolution of the fraction of open (unfouled) pores, $\mathcal{F}$, with a dimensionless backflush interval $t_{\mathrm{bf}}=1$ (solid) or $t_{\mathrm{bf}}=1 / 2$ (dashed). For sufficiently high frequencies of backflushing, the pores do not expand sufficiently to permit fouling (dashed blue curve). The inset shows a close-up of three backflushing cycles in which the elastic effects eventually cause fouling (only with the lower backflushing frequency).

a quasi-static elastic response of the two porous media. Various simplifications and approximations were made in the development of our model. For example, we neglected axial deformations caused by deformation and, in addition, any change in axial stress due to tangential stresses acting on the membrane wall. Our assumption of plane strain, $e_{z z}=0$, introduces an axial stress $\sigma_{z z}=v\left(\sigma_{r r}+\sigma_{\theta \theta}\right) \sim \mu Q \hat{d}_{\mathrm{m}} /\left(\hat{k}_{\mathrm{m}, 0} \hat{R}\right)$. The effect of viscous shear stresses, $\mu \partial u / \partial r$, acting on the wall is expected to lead to a change in the thickness-integrated tension along the length of the membrane $\Delta T=T(L)-T(0) \sim \mu Q L^{2} / \hat{R}^{4}$. For plane strain to be a realistic assumption, we require $\Delta T \ll \hat{d}_{\mathrm{m}} \sigma_{z z} \sim \mu Q \hat{d}_{\mathrm{m}}^{2} /\left(\hat{k}_{\mathrm{m}, 0} \hat{R}\right)$, which in turn requires

$$
\frac{\hat{k}_{\mathrm{m}, 0}}{\hat{d}_{\mathrm{m}}^{2}} \ll \frac{\hat{R}^{2}}{L^{2}} \ll 1,
$$

with the second inequality required to ensure a long, slender geometry. Given the typical values for microfiltration given in Appendix A, we find that this requirement is readily satisfied in practice.

Despite these simplifications, our model captures the advantages and disadvantages of elastic effects in membrane filtration (Figure 7). Initially, pores are expanded, which increases the flux of filtered water. However, with that comes increased deposition and irreversible fouling. In the long term, this increases fouling and operating pressures.

Despite the inevitable long-term consequences of elasticity, we can take advantage of the short-term reduction in pressure by considering our operating strategy. According to our model the backflush frequency may be tuned to ensure that the cake never grows thick enough to deform the membrane to the point where it fouls irreversibly (Figure 9). In this way, it should be possible to tune the operating conditions (particularly the operating flux and backflush frequency) as well as the membrane characteristics (permeability, pore size, material, etc.) to avoid fouling altogether. 
366 Nomenclature

Subscripts

$\mathrm{m}$

c

bf

0

1

Superscript

Dimensional
-

Symbols

E Young's modulus

$k \quad$ Permeability

$\kappa \quad$ Ratio of permeabilities

$\phi \quad$ Porosity

$v \quad$ Poisson ratio

$\mu \quad$ Viscosity

$Q \quad$ Flux

$\lambda \quad$ Particle deposition rate

$N_{0} \quad$ Initial number of open pores

$\beta \quad$ Cake layer to membrane thickness

$d \quad$ Layer thickness

$\mathcal{F} \quad$ Fouling

p Pressure

$\sigma \quad$ Stress

e Strain

$t \quad$ Time

$P \quad$ Driving pressure

$r \quad$ Radial coordinate

$u \quad$ Deformation

$A, B \quad$ Deformation parameters

$\Gamma, \gamma, \Omega, \omega \quad$ Deformation dimensionless numbers

$\Delta \quad$ Dilation

$a_{\text {pore }} \quad$ Pore radius

$a_{\text {part }} \quad$ Particle radius 


\section{Appendix A. Parameter estimates}

In the results of $\S 3$, we fix the parameter values of the system, unless otherwise stated. Our main purpose of that section is to understand whether the proposed mechanism (elasticityinduced pore expansion) works, so we do not compare a wide range of all the parameter values.

We take a uniform distribution of particles with sizes $4-5 \mu \mathrm{m}$, representative of microfiltration. To have maximum permeability while still rejecting particles, the undeformed pore size, $\hat{a}_{0}$, must be chosen to be as large as possible without allowing for fouling; we thus take $\hat{a}_{0}=3.957 \mu \mathrm{m}$. Specifically, we choose this $\hat{a}_{0}$ so that a small deformation may lead to fouling. Alternatively, for illustrative purposes we take a pore size of $\hat{a}_{0}=4.1 \mu \mathrm{m}$ so that $10 \%$ of particles can enter the membrane.

We take typical material parameter values for the undeformed state, which will also apply for the linearly elastic deformed state. We consider a tube with an internal radius $\hat{R}=10^{-3} \mathrm{~m}$ and membrane thickness $\hat{d}_{\mathrm{m}}=10^{-4} \mathrm{~m}$ [1] (i.e., a dimensionless membrane thickness $d_{\mathrm{m}}=10^{-1}$ ). The filtration velocity is of the order of millimetres to centimetres per second, say $0.5 \times 10^{-2} \mathrm{~m} / \mathrm{s}$, similar to those in a range of filtration experiments $[31,32]$. This corresponds to an areal flux through the annulus of $\hat{Q}=\pi \times 10^{-5} \mathrm{~m}^{2} / \mathrm{s}$,

The membrane properties are taken to be those of hollow-fibre membranes. The (undeformed) membrane permeability for microfiltration is of the order $\hat{k}_{\mathrm{m}, 0}=10^{-16} \mathrm{~m}^{2}$ [32]. Given the pore size and permeability, the porosity may be determined by relations such as KozenyCarman [28,30], giving $\varphi_{\mathrm{m}, 0} \simeq 0.35$. Typical values for the Young's modulus of membranes vary to a large degree, for example, of order $10^{8} \mathrm{~Pa}$ for ultrafiltration hollow fibre membranes [33] to order $10^{11} \mathrm{~Pa}$ for microfiltration membranes with a silicon-nitride layer [34]. Here we take the Young's modulus of the membrane, $\hat{E}_{\mathrm{m}}=10^{10} \mathrm{~Pa}$. We choose a Poisson ratio, $\nu_{\mathrm{m}}=0.3$, although a large range including negative values (e.g., honeycomb and carbon nanotube structures) are reported [34, 35, 36].

To the authors' knowledge, parameter values for the cake layer are limited. As such, the cake-layer properties are chosen for convenience, guided by some intuition. A value for cake permeability is difficult to determine as the cake layer is a collection of loose particles packed at the membrane surface, whose packing is non-trivial and beyond the scope of this work [15, 37]. Nevertheless, it is reasonable to consider a large permeability of the cake, $\hat{k}_{\mathrm{c}, 0}$ when caking is beginning, with smaller values later on when the cake is more compact. Since the cake layer represents a collection of particles of similar size to the membrane pore size, we assume that it has a similar (undeformed) permeability to the membrane, $\hat{k}_{\mathrm{c}, 0}=10^{-16} \mathrm{~m}^{2}$. We take the porosity, $\varphi_{\mathrm{c}, 0}=0.4$, to be slightly larger than the membrane as it is not a unified solid material. Analogously, we take the Young's modulus to be an order of magnitude lower than the membrane, $\hat{E}_{\mathrm{c}}=2 \times 10^{9} \mathrm{~Pa}$. Finally, we take the Poisson ratio to be lower than the membrane, $v_{\mathrm{c}}=0.2$ (which has been measured for a closely packed colloidal crystal, and has been used in the modelling of a colloidal gel [38]).

\section{Appendix B. Poroelastic Model}

In the context of poroelastic deformations, the fluid-structure interactions must be modelled in the same reference frame. However, it is usual to model the fluid flow in an Eulerian frame, and the solid mechanics in a Lagrangian frame. Assuming linear elasticity, the difference between these reference frames becomes negligible [39]. Further to this, in the context of linear elasticity, $\hat{R}$ is both the undeformed and deformed membrane radius. 


\section{Appendix B.1. Governing Equations}

A membrane tube or channel is usually long and thin, resulting in a small reduced Reynolds number [40]. Assuming that the flow is quasi-steady, we use the steady thin-layer Stokes equations (or lubrication equations) for the flow inside the tube.

The fluid flow within the central void of the 2D annular cross-section of the filter (Figure 3 ) is given by the axisymmetric Stokes equations

$$
\begin{gathered}
\frac{1}{\hat{r}} \frac{\partial}{\partial \hat{r}}(\hat{r} \hat{V})=0 \\
\frac{\hat{\mu}}{\hat{r}} \frac{\partial}{\partial \hat{r}}\left(\hat{r} \frac{\partial \hat{V}}{\partial \hat{r}}\right)=\frac{\partial \hat{p}}{\partial \hat{r}},
\end{gathered}
$$

where $\hat{V}$ is the radial velocity, $\hat{p}$ is the radial pressure, and $\hat{\mu}$ is the fluid viscosity. The flow in the porous cake layer and membrane is given by Darcy's equation in polar coordinates

$$
\hat{V}=-\frac{\hat{k}}{\hat{\mu}} \frac{\partial \hat{p}}{\partial \hat{r}},
$$

where $\hat{k}$ is the permeability of the porous medium. The pressure, $\hat{p}$, and velocity, $\hat{V}$, are continuous at the cake-layer-membrane boundary. We note that the fluid velocity in the porous media is the Darcy velocity and depends on the porosity of each medium. It is this velocity that is assumed to be continuous at each interface. We consider the case of a constant flux $\hat{Q}$ passing through the system. The reference pressure outside the membrane is taken to be zero.

For the setup of a 2D annulus with constant influx (Figure 3), the pressure is constant in the internal void, consistent with thin-layer Stokes flow [41]. However, a pressure difference, the transmembrane pressure difference (TMP), exists across the membrane driving the flow. The stress from the fluid pressure deforms the membrane and cake elastically.

The steady-state linear elastic deformation of a solid material is governed by the steady Navier-Cauchy equation with no body forces [29],

$$
\hat{\nabla} \cdot \hat{\sigma}^{\mathrm{t}}=0
$$

where $\hat{\nabla}$ is the dimensional gradient operator and $\hat{\sigma}^{t}$ is the total stress in the material. For a poroelastic material, the total stress is often given according to Terzaghi's principle [27, 28] by

$$
\hat{\sigma}_{i j}^{\mathrm{t}}=\hat{\sigma}_{i j}-\hat{p} \delta_{i j}
$$

where $\hat{\sigma}$ is the elastic stress of the material (due to strain), and $-\hat{p} \mathbf{I}$ is the contribution due to the fluid pore pressure (where I denotes the identity matrix). Hence, the excess of stress over pore pressure drives the deformation.

The linearly elastic deformations are modelled using plane strain, whereby the elastic stress components may be written in terms of the deformation, $\hat{u}$, as

$$
\begin{aligned}
& \hat{\sigma}_{r r}=\frac{\hat{E}}{(1+v)(1-2 v)}\left((1-v) \frac{\mathrm{d} \hat{u}}{\mathrm{~d} \hat{r}}+v \frac{\hat{u}}{\hat{r}}\right), \\
& \hat{\sigma}_{\theta \theta}=\frac{\hat{E}}{(1+v)(1-2 v)}\left(v \frac{\mathrm{d} \hat{u}}{\mathrm{~d} \hat{r}}+(1-v) \frac{\hat{u}}{\hat{r}}\right),
\end{aligned}
$$


where $\hat{E}$ is the Young's modulus and $v$ is the Poisson ratio [29]. We use subscripts $m$ and $c$ to denote values in the membrane or cake respectively. For the full membrane tube, plane strain would model the case of constant TMP, i.e., constant permeate flux along the membrane, as found in direct-flow filtration [26].

\section{Appendix B.2. Non-dimensionalization}

We non-dimensionalize using the following scalings

$$
\begin{aligned}
& \hat{r}=\hat{R} r, \quad \hat{u}_{\mathrm{m}}^{0}=\hat{R} u_{\mathrm{m}}^{0}, \quad \hat{u}_{\mathrm{m}}=\hat{R} u_{\mathrm{m}}, \quad \hat{u}_{\mathrm{c}}=\hat{R} u_{\mathrm{c}}, \quad \hat{V}=\frac{\hat{Q}}{\hat{R}} V, \quad \hat{d}_{\mathrm{c}}=\hat{R} d_{\mathrm{c}}, \\
& \hat{d}_{\mathrm{m}}=\hat{R} d_{\mathrm{m}}, \quad \hat{p}=\frac{\hat{Q} \hat{\mu}}{\hat{k}_{\mathrm{m}, 0}} p, \quad \hat{P}_{1}=\frac{\hat{Q} \hat{\mu}}{\hat{k}_{\mathrm{m}, 0}} P_{1}, \quad \hat{P}_{2}=\frac{\hat{Q} \hat{\mu}}{\hat{k}_{\mathrm{m}, 0}} P_{2}, \quad \hat{\sigma}_{r r}=\hat{P}_{1} \sigma_{r r}, \quad \hat{\sigma}_{\theta \theta}=\hat{P}_{1} \sigma_{\theta \theta} .
\end{aligned}
$$

Here, $\hat{u}_{\mathrm{m}}^{0}$ is the membrane deformation in the absence of any cake layer, $\hat{u}_{\mathrm{m}}$ and $\hat{u}_{\mathrm{c}}$ are the deformations of the membrane and the cake during operation, respectively, $\hat{d}_{\mathrm{m}}$ and $\hat{d}_{\mathrm{c}}$ are the thickness of the membrane and cake layer respectively, $\hat{P}_{1}$ is the initial pressure on the inner membrane surface, and $P_{2}$ is the initial pressure on the cake inner surface.

The problem is scaled with the material parameters of the membrane as this is a well-defined material, unlike the cake. We assume that the Young's modulus, $\hat{E}$, and Poisson ratio, $v$, of both the membrane and cake layer, and the permeability of the cake layer, remain constant to leading order (deformation enters only at higher order).

As the membrane is deformed, both the pore size and porosity change. However, as the porosity is reduced due to membrane fouling, characterized by the density of open pores, $\mathcal{F}$, we assume that the dimensionless membrane permeability, $k_{\mathrm{m}}$, decreases uniformly. Both deformation and fouling contribute to the change in membrane structure, and hence, permeability. We write the dimensionless permeability, $k_{\mathrm{m}}$, as

$$
k_{\mathrm{m}}=\left(k_{\mathrm{m}, 0}+k_{\mathrm{m}, 1}\right) \mathcal{F},
$$

where $k_{\mathrm{m}, 0}$ is the leading order undeformed permeability and $k_{\mathrm{m}, 1}$ is the effect of a small elastic deformation on the permeability.

\section{Appendix B.3. Fluid Flow}

The leading-order (undeformed) solution to the 2D symmetric radial fluid-flow problem can be written down immediately as

$$
\begin{aligned}
& V=\frac{1}{2 \pi r}, \\
& p= \begin{cases}\frac{1}{2 \pi \mathcal{F}} \log \left(1+d_{\mathrm{m}}\right)-\frac{1}{2 \pi \kappa} \log \left(1-d_{\mathrm{c}}\right), & r<1-d_{\mathrm{c}}, \\
-\frac{1}{2 \pi \kappa} \log r+\frac{1}{2 \pi \mathcal{F}} \log \left(1+d_{\mathrm{m}}\right), & 1-d_{\mathrm{c}}<r<1, \\
-\frac{1}{2 \pi \mathcal{F}} \log r+\frac{1}{2 \pi \mathcal{F}} \log \left(1+d_{\mathrm{m}}\right), & 1<r<1+d_{\mathrm{m}}, \\
0, & r>1+d_{\mathrm{m}},\end{cases}
\end{aligned}
$$


where

$$
\kappa=\frac{\hat{k}_{\mathrm{c}, 0}}{\hat{k}_{\mathrm{m}, 0}},
$$

${ }_{443}$ is the ratio of undeformed dimensional permeabilities between the cake and membrane.

The radial pressure, $\hat{P}$, inside the void that drives the flow is constant in space, as expected for flow in a thin channel, and so equals the pressure at the internal-cake interface. However, it is time-dependent via $\mathcal{F}$ and $d_{\mathrm{c}}$ as described by (8). The dimensionless reference pressures, $\hat{P}_{1}$ at the membrane-cake-layer interface $(r=1)$, and $\hat{P}_{2}$ at the internal-cake interface $\left(r=1-d_{\mathrm{c}}\right)$, are given by

$$
\begin{aligned}
& \hat{P}_{1}=\frac{1}{2 \pi \mathcal{F}} \log \left(1+d_{\mathrm{m}}\right), \\
& \hat{P}_{2}=\frac{1}{2 \pi \mathcal{F}} \log \left(1+d_{\mathrm{m}}\right)-\frac{1}{2 \pi \kappa} \log \left(1-d_{\mathrm{c}}\right) .
\end{aligned}
$$

\section{Appendix B.4. Deformation}

When the filtration process begins (originally or after a backflush) there is no cake layer present. The membrane deformation, $u_{\mathrm{m}}^{0}$, is given by the dimensionless version of the NavierCauchy equation (B.3), with boundary conditions given by the (plane strain) stresses (B.5) on the inner $(r=1)$ and outer $\left(r=1+d_{\mathrm{c}}\right)$ walls of the membrane; that is, the fluid pressure at the boundaries,

$$
\begin{aligned}
\frac{\mathrm{d}}{\mathrm{d} r}\left(\frac{1}{r} \frac{\mathrm{d}}{\mathrm{d} r}\left(r u_{\mathrm{m}}^{0}\right)\right)=\frac{\Gamma}{\Omega} \frac{\mathrm{d} p}{\mathrm{~d} r} & =-\frac{\Gamma}{\Omega} \frac{1}{2 \pi \mathcal{F} r} \quad\left(1<r<1+d_{\mathrm{m}}\right), \\
\left.\Omega\left(\left(1-v_{\mathrm{m}}\right) \frac{\mathrm{d} u_{\mathrm{m}}^{0}}{\mathrm{~d} r}+v_{\mathrm{m}} \frac{u_{\mathrm{m}}^{0}}{r}\right)\right|_{r=1} & =-1, \\
\left.\Omega\left(\left(1-v_{\mathrm{m}}\right) \frac{\mathrm{d} u_{\mathrm{m}}^{0}}{\mathrm{~d} r}+v_{\mathrm{m}} \frac{u_{\mathrm{m}}^{0}}{r}\right)\right|_{r=1+d_{\mathrm{m}}} & =0,
\end{aligned}
$$

with $p$ given in (B.8b), and

$$
\begin{array}{r}
\Omega=\frac{\hat{E}_{\mathrm{m}}}{\hat{P}_{1}\left(1+v_{\mathrm{m}}\right)\left(1-2 v_{\mathrm{m}}\right)}, \\
\Gamma=\frac{2 \pi}{\left(1-v_{\mathrm{m}}\right) \log \left(1+d_{\mathrm{m}}\right)},
\end{array}
$$

are dimensionless parameters that apply (for reference) to the unfouled $(\mathcal{F}=1)$ membrane; $\Omega$ is a parameter related to the physics of the system, representing a stiffness since $\hat{E}_{\mathrm{m}} / \hat{P}_{1} \propto \hat{E}_{\mathrm{m}} / \hat{Q} ; \Gamma$ is a purely geometrical parameter.

The solution to (B.11) for $u_{\mathrm{m}}^{0}$ reads

$$
u_{\mathrm{m}}^{0}=-\frac{\Gamma}{8 \pi \Omega \mathcal{F}}(-r+2 r \log r)+A_{\mathrm{m}}^{0} r+\frac{B_{\mathrm{m}}^{0}}{r},
$$


for $1<r<1+d_{\mathrm{m}}$, where

$$
\begin{aligned}
A_{\mathrm{m}}^{0} & =\frac{8 \pi \mathcal{F}+\Gamma\left\{\left[\left(1+d_{\mathrm{m}}\right)^{2}-1\right]\left(1-2 v_{\mathrm{m}}\right)+2\left(1+d_{\mathrm{m}}\right)^{2} \log \left(1+d_{\mathrm{m}}\right)\right\}}{8 \pi \mathcal{F} \Omega\left[\left(1+d_{\mathrm{m}}\right)^{2}-1\right]}, \\
B_{\mathrm{m}}^{0} & =\frac{\left(1+d_{\mathrm{m}}\right)^{2}\left[4 \pi \mathcal{F}+\Gamma \log \left(1+d_{\mathrm{m}}\right)\right]}{4 \pi \mathcal{F} \Omega\left(1-2 v_{\mathrm{m}}\right)\left[\left(1+d_{\mathrm{m}}\right)^{2}-1\right]} .
\end{aligned}
$$

When a cake layer is present, the deformation of this layer, $u_{\mathrm{c}}$, and membrane deformation, $u_{\mathrm{m}}$, are given by a set of Navier-Cauchy equations (B.3). As the cake layer is retained by the membrane, continuity of stress holds at the cake-membrane interface. Since the fluid stress is continuous here, the elastic stress must also be continuous. Furthermore, we must have continuity of the deformation at the cake-membrane interface. However, before the cake layer was deposited, the membrane was deformed by $u_{\mathrm{m}}^{0}$. We therefore impose continuity of additional deformation once the cake is deposited (i.e., $u_{\mathrm{c}}=u_{\mathrm{m}}-u_{\mathrm{m}}^{0}$ at the cake-membrane interface). The remaining boundary conditions are again due to the pressure on the inner- and outer-most surfaces. These conditions are written as

$$
\begin{aligned}
\frac{\mathrm{d}}{\mathrm{d} r}\left(\frac{1}{r} \frac{\mathrm{d}}{\mathrm{d} r}\left(r u_{\mathrm{m}}\right)\right) & =\frac{\Gamma}{\Omega} \frac{\mathrm{d} p}{\mathrm{~d} r}=-\frac{\Gamma}{\Omega} \frac{1}{2 \pi \mathcal{F} r} \quad\left(1<r<1+d_{\mathrm{m}}\right), \\
\frac{\mathrm{d}}{\mathrm{d} r}\left(\frac{1}{r} \frac{\mathrm{d}}{\mathrm{d} r}\left(r u_{\mathrm{c}}\right)\right) & =\frac{\Gamma}{\Omega} \frac{\gamma}{\omega} \frac{\mathrm{d} p}{\mathrm{~d} r}=-\frac{\Gamma}{\Omega} \frac{\gamma}{\omega} \frac{1}{2 \pi \kappa r} \quad\left(1-d_{\mathrm{c}}<r<1\right), \\
\left.\Omega \omega\left(\left(1-v_{\mathrm{c}}\right) \frac{\mathrm{d} u_{\mathrm{c}}}{\mathrm{d} r}+v_{\mathrm{c}} \frac{u_{\mathrm{c}}}{r}\right)\right|_{r=1-d_{\mathrm{c}}} & =-\frac{\hat{P}_{2}}{\hat{P}_{1}}=-\left(1-\frac{\mathcal{F}}{\kappa} \frac{\log \left(1-d_{\mathrm{c}}\right)}{\log \left(1+d_{\mathrm{m}}\right)}\right), \\
\left.\Omega\left(\left(1-v_{\mathrm{m}}\right) \frac{\mathrm{d} u_{\mathrm{m}}}{\mathrm{d} r}+v_{\mathrm{m}} \frac{u_{\mathrm{m}}}{r}\right)\right|_{r=1+d_{\mathrm{m}}} & =0, \\
\left.\omega\left(\left(1-v_{\mathrm{c}}\right) \frac{\mathrm{d} u_{\mathrm{c}}}{\mathrm{d} r}+v_{\mathrm{c}} \frac{u_{\mathrm{c}}}{r}\right)\right|_{r=1} & =\left.\left(\left(1-v_{\mathrm{m}}\right) \frac{\mathrm{d} u_{\mathrm{m}}}{\mathrm{d} r}+v_{\mathrm{m}} \frac{u_{\mathrm{m}}}{r}\right)\right|_{r=1}, \\
u_{\mathrm{c}}(r=1) & =u_{\mathrm{m}}(r=1)-u_{\mathrm{m}}^{0}(r=1),
\end{aligned}
$$

where $p$ is given in (B.8b), and the dimensionless parameters

$$
\begin{aligned}
\omega & =\frac{\hat{E}_{\mathrm{c}}}{\left(1+v_{\mathrm{c}}\right)\left(1-2 v_{\mathrm{c}}\right)} / \frac{\hat{E}_{\mathrm{m}}}{\left(1+v_{\mathrm{m}}\right)\left(1-2 v_{\mathrm{m}}\right)}, \\
\gamma & =\frac{1-v_{\mathrm{m}}}{1-v_{\mathrm{c}}},
\end{aligned}
$$

reflect the material parameter ratios between the cake layer and membrane.

The solution to (B.15) for $u_{\mathrm{m}}$ and $u_{\mathrm{c}}$ reads

$$
\begin{array}{ll}
u_{\mathrm{m}}=-\frac{\Gamma}{8 \pi \Omega \mathcal{F}}(-r+2 r \log r)+A_{\mathrm{m}} r+\frac{B_{\mathrm{m}}}{r}, & 1<r<1+d_{\mathrm{m}}, \\
u_{\mathrm{c}}=-\frac{\Gamma \gamma}{8 \pi \Omega \omega \kappa}(-r+2 r \log r)+A_{\mathrm{c}} r+\frac{B_{\mathrm{c}}}{r}, & 1-d_{\mathrm{c}}<r<1,
\end{array}
$$

where $A_{\mathrm{m}}, B_{\mathrm{m}}, A_{\mathrm{c}}$, and $B_{\mathrm{c}}$ are constants determined by substituting the general solutions (B.17) into the boundary conditions (B.15c-f). These constants may be determined explicitly, but for 
brevity we show their expressions in matrix form,

$$
\left(\begin{array}{c}
A_{\mathrm{m}} \\
B_{\mathrm{m}} \\
A_{\mathrm{c}} \\
B_{\mathrm{c}}
\end{array}\right)=\left(\begin{array}{cccc}
\left(1+d_{\mathrm{m}}\right)^{2} & -1+2 v_{\mathrm{m}} & 0 & 0 \\
0 & 0 & \left(1-d_{\mathrm{c}}\right)^{2} & -1+2 v_{\mathrm{c}} \\
1 & -1+2 v_{\mathrm{m}} & -\omega & \omega\left(1-2 v_{\mathrm{c}}\right) \\
-1 & -1 & 1 & 1
\end{array}\right)^{-1} \Psi
$$

where

$$
\Psi=\left(\begin{array}{c}
\frac{\Gamma\left(1+d_{\mathrm{m}}\right)^{2}}{8 \pi \mathcal{F} \Omega}\left[1-2 v_{\mathrm{m}}+2 \log \left(1+d_{\mathrm{m}}\right)\right] \\
\frac{\left(1-d_{\mathrm{c}}\right)^{2}}{8 \pi \kappa \Omega \omega}\left[-8 \pi \kappa+\Gamma \gamma\left(1-2 v_{\mathrm{c}}\right)+2 \frac{\log \left(1-d_{\mathrm{c}}\right)}{\log \left(1+d_{\mathrm{m}}\right)}\left(4 \pi \mathcal{F}+\Gamma \gamma \log \left(1+d_{\mathrm{m}}\right)\right)\right] \\
\frac{\Gamma}{8 \pi \kappa \Omega}\left[\gamma\left(-1+2 v_{\mathrm{c}}\right)+\frac{\kappa\left(1-2 v_{\mathrm{m}}\right)}{\mathcal{F}}\right] \\
-u_{\mathrm{m}}^{0}(1)+\frac{\gamma}{8 \pi \Omega}\left[\frac{1}{\mathcal{F}}-\frac{\gamma}{\kappa \omega}\right]
\end{array}\right) .
$$

When the cake thickness is zero, $A_{\mathrm{m}}$ and $B_{\mathrm{m}}$ reduce to (B.14), obtained in the absence of a cake. We note that the solution incorporates the deformation with no cake layer, $u_{\mathrm{m}}^{0}(\mathrm{~B} .13)$, after the last backflush or at the beginning of the experiment.

[1] G. K. Pearce, UF/MF Membrane Water Treatment: Principles and Design, Water Treatment Academy, 2011.

[2] N. Delgrange-Vincent, C. Cabassud, M. Cabassud, L. Durand-Bourlier, J. M. Laine, Neural networks for long term prediction of fouling and backwash efficiency in ultrafiltration for drinking water production, Desalination 131 (2000) 353-362.

[3] P. J. Smith, S. Vigneswaran, H. H. Ngo, R. Ben-Aim, H. Nguyen, A new approach to backwash initiation in membrane systems, Journal of Membrane Science 278 (2006) 381-389.

[4] Y. Ye, V. Chen, P. Le-Clech, Evolution of fouling deposition and removal on hollow fibre membrane during filtration with periodical backwash, Desalination 283 (2011) 198-205.

[5] J. Cakl, I. Bauer, P. Doleček, P. Mikulášek, Effects of backflushing conditions on permeate flux in membrane crossflow microfiltration of oil emulsion, Desalination 127 (2000) 189-198.

[6] S. Hong, P. Krishna, C. Hobbs, D. Kim, J. Cho, Variations in backwash efficiency during colloidal filtration of hollow-fiber microfiltration membranes, Desalination 173 (2005) 257-268.

[7] P. J. Remize, C. Guigui, C. Cabassud, Evaluation of backwash efficiency, definition of remaining fouling and characterisation of its contribution in irreversible fouling: Case of drinking water production by air-assisted ultrafiltration, Journal of Membrane Science 355 (2010) 104-111.

[8] Q. She, X. Jin, C. Y. Tang, Osmotic power production from salinity gradient resource by pressure retarded osmosis: Effects of operating conditions and reverse solute diffusion, Journal of Membrane Science 401 (2012) 262-273.

[9] J. G. Herterich, I. M. Griffiths, R. W. Field, D. Vella, The effect of a concentration-dependent viscosity on particle transport in a channel flow with porous walls, AIChE Journal 60 (2014) 1891-1904.

[10] R. W. Field, J. J. Wu, Modelling of permeability loss in membrane filtration: Re-examination of fundamental fouling equations and their link to critical flux, Desalination 283 (2011) 68-74.

[11] I. M. Griffiths, A. Kumar, P. S. Stewart, A combined network model for membrane fouling, Journal of Colloid and Interface Science 432 (2014) 10-18.

[12] J. Hermia, Constant pressure blocking filtration laws - application to power-law non-Newtonian fluids, Transactions of the Institution of Chemical Engineers 60 (1982) 183-187.

[13] C.-C. Ho, A. L. Zydney, A combined pore blockage and cake filtration model for protein fouling during microfiltration, Journal of Colloid and Interface Science 232 (2000) 389-399.

[14] W. R. Bowen, F. Jenner, Theoretical descriptions of membrane filtration of colloids and fine particles: an assessment and review, Advances in Colloid and Interface Science 56 (1995) 141-200. 
[15] K.-J. Hwang, Y.-S. Wu, W.-M. Lu, The surface structure of cake formed by uniform-sized rigid spheroids in cake filtration, Powder technology 87 (1996) 161-168.

[16] S. S. Madaeni, The application of membrane technology for water disinfection, Water Research 33 (1999) 301-308.

[17] S. B. Sadr Ghayeni, P. J. Beatson, A. G. Fane, R. P. Schneider, Bacterial passage through microfiltration membranes in wastewater applications, Journal of Membrane Science 153 (1999) 71-82.

[18] Q. T. Nguyen, J. Neel, Characterization of ultrafiltration membranes.: Part iv. influence of the deformation of macromolecular solutes on the transport through ultrafiltration membranes., Journal of Membrane Science 14 (1983) 111-127.

[19] S. Chou, R. Wang, A. G. Fane, Robust and high performance hollow fiber membranes for energy harvesting from salinity gradients by pressure retarded osmosis, Journal of Membrane Science 448 (2013) 44-54.

[20] B. Van der Bruggen, C. Vandecasteele, T. Van Gestel, W. Doyen, R. Leysen, A review of pressure-driven membrane processes in wastewater treatment and drinking water production, Environmental Progress 22 (2003) 46-56.

[21] W. Guo, H.-H. Ngo, J. Li, A mini-review on membrane fouling, Bioresource Technology 122 (2012) $27-34$.

[22] K.-J. Hwang, C.-L. Hsueh, Dynamic analysis of cake properties in microfiltration of soft colloids, Journal of Membrane Science 214 (2003) 259-273.

[23] J. Altmann, S. Ripperger, Particle deposition and layer formation at the crossflow microfiltration, Journal of Membrane Science 124 (1997) 119-128.

[24] L. Pocivavsek, J. Pugar, R. O’Dea, S.-H. Ye, W. Wagner, E. Tzeng, S. Velankar, E. Cerda, Topography-driven surface renewal, Nature Physics (2018) https://doi.org/10.1038/s41567-018-0193-X.

[25] Y. K. Benkahla, A. Ould-Dris, M. Y. Jaffrin, D. Si-Hassen, Cake growth mechanism in cross-flow microfiltration of mineral suspensions, Journal of Membrane Science 98 (1995) 107-117.

[26] J. G. Herterich, Q. Xu, R. W. Field, D. Vella, I. M. Griffiths, Optimizing the operation of a direct-flow filtration device, Journal of Engineering Mathematics 104 (2016) 195-211.

[27] C. W. MacMinn, E. R. Dufresne, J. S. Wettlaufer, Fluid-driven deformation of a soft granular material, Physical Review X 5 (2015) 011020.

[28] O. Coussy, Mechanics and Physics of Porous Solids, John Wiley \& Sons, 2011.

[29] P. Howell, G. Kozyreff, J. R. Ockendon, Applied Solid Mechanics, 43, Cambridge University Press, 2009.

[30] P. Xu, B. Yu, Developing a new form of permeability and Kozeny-Carman constant for homogeneous porous media by means of fractal geometry, Advances in Water Resources 31 (2008) 74-81.

[31] A. Gabelman, S.-T. Hwang, Hollow fiber membrane contactors, Journal of Membrane Science 159 (1999) 61-106

[32] R. J. Shipley, S. L. Waters, M. J. Ellis, Definition and Validation of Operating Equations for Poly(Vinyl Alcohol)Poly(Lactide-Co-Glycolide) Microfiltration Membrane-Scaffold Bioreactors, Biotechnology and Bioengineering 107 (2010) 382-392.

[33] J. Qin, T.-S. Chung, Effect of dope flow rate on the morphology, separation performance, thermal and mechanical properties of ultrafiltration hollow fibre membranes, Journal of Membrane Science 157 (1999) 35-51.

[34] C. J. Rijn, M. C. Elwenspoek, Micro filtration membrane sieve with silicon micro machining for industrial and biomedical applications (1995)

[35] H. Ma, J. Zeng, M. L. Realff, S. Kumar, D. A. Schiraldi, Processing, structure, and properties of fibers from polyester/carbon nanofiber composites, Composites Science and Technology 63 (2003) 1617-1628.

[36] Y. J. Ma, X. F. Yao, Q. S. Zheng, Y. J. Yin, D. J. Jiang, G. H. Xu, F. Wei, Q. Zhang, Carbon nanotube films change Poissons ratios from negative to positive, Applied Physics Letters 97 (2010) 061909.

[37] J. Mendret, C. Guigui, P. Schmitz, C. Cabassud, In situ dynamic characterisation of fouling under different pressure conditions during dead-end filtration: compressibility properties of particle cakes, Journal of Membrane Science 333 (2009) 20-29.

[38] R. W. Style, S. S. L. Peppin, Crust formation in drying colloidal suspensions, Proceedings of the Royal Society of London A: Mathematical, Physical and Engineering Sciences 467 (2011) 174-193.

[39] C. W. MacMinn, E. R. Dufresne, J. S. Wettlaufer, Large deformations of a soft porous material, Physical Review Applied 5 (2016) 044020(30).

[40] J. G. Herterich, D. Vella, R. W. Field, N. P. Hankins, I. M. Griffiths, Tailoring wall permeabilities for enhanced filtration, Physics of Fluids 27 (2015) 053102.

[41] O. Reynolds, On the theory of lubrication and its application to Mr. Beauchamp Tower's experiments, including an experimental determination of the viscosity of olive oil., Proceedings of the Royal Society of London 40 (1886) 191-203. 